\title{
Isogeometric collocation for three-dimensional geometrically exact shear-deformable beams
}

\author{
Enzo Marino* \\ Department of Civil and Environmental Engineering, University of Florence, Via di S. Marta 3, 50139 Firenze, Italy
}

Received 23 January 2016; received in revised form 13 April 2016; accepted 15 April 2016

Available online 27 April 2016

\section{Highlights}

- Isogeometric collocation is extended to geometrically exact beams.

- Consistent linearization of the strong form of the governing equations is derived.

- Incremental rotations are parametrized through Eulerian rotation vectors.

- Numerical tests show efficiency and high accuracy.

\begin{abstract}
We extend the isogeometric collocation method to the geometrically nonlinear beams. An exact kinematic formulation, able to represent three-dimensional displacements and rotations without any restriction in magnitude, is presented without the introduction of the moving frame concept. A displacement-based formulation is adopted. Full linearization of the strong form of the governing equations is derived consistently with the underlying geometric structure of the configuration manifold. Incremental rotations are parametrized through Eulerian rotation vectors and configuration updates are performed by means of the exponential map. Numerical tests demonstrate that the proposed combination of isogeometric collocation method with the chosen rotations parametrization results in an efficient computational scheme able to model complex problems with high accuracy.
\end{abstract}

(C) 2016 Elsevier B.V. All rights reserved.

Keywords: Isogeometric collocation; Geometrically exact beams; Shear-deformable nonlinear beams; Finite strains; Finite rotations; SO(3)

\section{Introduction}

Beam-like structures undergoing very large three-dimensional displacements and rotations are routinely required to model a large variety of engineering problems. Motivated by the need to reduce computational cost ensuring high accuracy levels, and to develop flexible tools capable of representing complex geometries exactly, this paper represents a first step towards the extension of isogeometric collocation (IGA-C) methods to geometrically exact

\footnotetext{
* Tel.: +39055 2758858 .

E-mail address: enzo.marino@dicea.unifi.it.
} 
reduced-dimensional continua. With "three-dimensional geometrically exact" we intend a kinematic model able to describe motion and strains without any restriction or approximation.

Isogeometric analysis (IGA) is a method for the solution of problems governed by partial differential equations introduced in 2005 by Hughes et al. [1]. The primary goals of IGA are to represent the exact geometry no matter how coarse the discretization and to simplify the expensive mesh generation and refinement processes required by traditional Finite Element Analysis (FEA). IGA achieves these goals by using the functions adopted in Computer Aided Design (CAD) not only to describe the domain geometry, but also to represent the numerical solution of the differential problem, filling the traditional gap between CAD and Finite Element (FE) solvers. A complete exposition of IGA can be found in the book [2].

Over the last decade, IGA has become a well-established method used in a wide range of problems including structural mechanics [3-12] with recent developments for spatial Bernoulli beams [13,14], turbulent flow and fluid-structure interaction [15-20].

In IGA, the smooth higher-order basis functions raise the need to develop more efficient quadrature rules [21,22]. In fact, element-wise Gauss quadrature is considered optimal for standard FEA but sub-optimal for IGA, since it does not exploit the inter-element continuity of the smooth basis functions.

IGA-C is a new promising method introduced in [23] based on the discretization of the strong form of the governing equations. The method takes advantage of the higher-continuity properties of the approximating functions adopted in IGA and permits reducing significantly the computational costs by eliminating the need for numerical quadrature [24]. A major advantage of the collocation method over Galerkin-based formulations is that only one point evaluation (collocation point) per element is required. This makes the method very attractive especially for those applications where efficiency is directly related to the cost of the integration over the elements. A comprehensive investigation about the advantages of IGA-C over Galerkin-based methods is reported in [25], where it is shown that IGA-C can be orders of magnitude faster than Galerkin-based IGA and Galerkin-based FEA to achieve a specified level of accuracy. In [26] the IGA-C method is further developed and extended to the case of explicit dynamics.

In the framework of one-dimensional continua, applications of IGA-C to initially straight planar and threedimensional curved Timoshenko beams are proposed in [27] and [28], respectively. In both papers it is demonstrated numerically and theoretically that mixed IGA-C formulations are locking-free for any choice of the discrete spaces for displacements, rotations, and internal forces. An IGA-C approach for Bernoulli-Euler beams and Kirchhoff plates has been proposed in [29], where accuracy, stability, and robustness of the method are shown. A further development of the method is presented in [30], where isogeometric collocation methods for Reissner-Mindlin plates are proposed for the first time. IGA-C has been used to successfully solve a new single-variable formulation for shear-deformable beams recently introduced in [31]. Two important aspects in the development of isogeometric collocation, that are the imposition of Neumann boundary conditions and the contact constraints between multiple patches, are addressed in [32].

All the works briefly recalled above are based on linear elastic models. Kruse et al. [33] have been the first to study the performance of IGA-C in the case of fully nonlinear problems, including both geometrical and mechanical nonlinearities. In particular the authors have explored the application of IGA-C to large deformation elasticity and frictional contact problems. They have shown that in the context of large deformation elasticity, collocation offers substantial benefits especially in the three-dimensional case and for higher discretization orders.

For a large variety of engineering problems reduced-dimension continua, like beams and shells, represent a more convenient choice than three-dimensional models. This point, along with the distinctive computational features of IGA-C summarized above, motivate and suggest initiating a research path oriented to the extension of IGA-C to geometrically exact rods which, so far, is an unexplored field.

The first complete theoretical development of a finite strain beam model was given by Simo in 1985 [34]. In that paper the author generalized to the three-dimensional dynamic case the rod models earlier proposed in [35,36]. In these works, the Euler-Bernoulli hypothesis, for which there can be no shears of the beam cross section with respect to the axis, is removed and it is assumed that the cross section remains planar during the deformation but not necessarily orthogonal to the rod axis (Timoshenko-Reissner model). The underlying kinematic models in $[34,36]$ were developed within the context of director type reduced continua. The concept of "directed" continua was first systematically developed in 1909 by E. and F. Cosserat, (we refer to [37] for a review up to 1981). Within this kinematic framework, the rod motion is described by means of a three-dimensional moving frame, which is an orthogonal moving basis defined in such a way that one of its vector is orthogonal to the rod cross section in any configuration. The evolution 
of the moving frame is tracked through a space-time-dependent orthogonal matrix called rotation operator [38]. A recent review of the beam theories in the language of geometric mechanics can be found in [39].

A FE formulation of the governing equations derived in [34] was developed in [40] for the static case. The distinctive novelty was the procedure employed to update the rotation operator based on the superimposition of the incremental rotation, represented by a skew-symmetric tensor field, to the current rotation. In [40], the implementation of the update procedure was based on the use of quaternion parameters. A more recent insight into the kinematics of the Simo's beam theory [34] is given in [41].

The rotation operator and its updating procedure play a crucial role in the theoretical and computational development of geometrically exact kinematic beams. The rotation operator belongs to the Special Orthogonal group $\mathrm{SO}(3)$, which is a non-commutative Lie group. $\mathrm{SO}(3)$ is a nonlinear differential manifold $[42,43]$ on which standard concepts like addition and linearization become meaningless in a global sense. An additional geometric structure, named tangent space, is required to approximate locally the nonlinear manifold with a vector space, where standard operations can be performed. The local nature of the tangent space represents the main complexity behind the numerical schemes because objects belonging to different tangent spaces, such as rotation increments, cannot be directly summed. A detailed discussion about the rotation manifold $\mathrm{SO}(3)$ and its tangent spaces is offered by Mäkinen in $[44,45]$.

A fundamental contribution to the development of geometrically exact beam theories came in 1988 by Cardona and Geradin [46]. They introduced a linear mapping, sometimes refereed to as tangential transformation, in order to project the rotation increments belonging to different tangent spaces onto the same tangent space where standard operations make sense. Different update procedures named Eulerian, total Lagrangian and updated Lagrangian were defined in [46]. In the total Lagrangian formulation, adopted also in [47-49], the rotation vector is used to represent the total rotation between initial and current configurations resulting, as highlighted in [48], in a full vector-like parametrization of the rotation operator. The total Lagrangian formulation has two main disadvantages: (i) it suffers singularity problems at rotation angle $2 \pi$ and its multiples and (ii) it presents difficulties to obtain full linearized equations, see for example appendix of [47]. The latter drawback is addressed by Ritto-Corrêa and Camotim in [48], where second-order directional derivatives of the kinematic operators are provided and a simpler expression for the tangent operator is formulated. The former is addressed by Ibrahimbegovic in [50] where, as opposed to formulations where vector parameters are defined in the global sense, an incremental vector-like rotation parameter is used. The main advantage of such a choice is not only the symmetric form of the tangent operator, but also the singularity of the vector-like parametrization is avoided. Mäkinen [49] proposed a total Lagrangian formulation able to bypass the singularity by introducing a change of parametrization on the rotation manifold.

In this work we adopt a formulation in which incremental rotations are parametrized by means of Eulerian (spatial) rotation vectors. Configuration updates are made directly by exponentiating and superimposing the incremental rotation to the current rotation as in [50]. Incremental rotations are skew-symmetric tensors associated to the Eulerian rotation vectors. The update operation crucially relies on the Rodrigues formula (see [51] for a discussion about the origins of the formula) which represents the exact expression of the exponential map for $\mathrm{SO}(3)$. The incremental rotation vectors, together with the beam axis displacements, are assumed as independent kinematic variables in our displacement-based formulation. Unlike [40,52], no use of quaternion parameters is made.

In the present formulation, issues related to the exact differentiation of the tangential transformation (typically involving cumbersome calculations) $[48,53]$ are avoided, and the reparametrization of the rotation operator on the complement rotation angle $[49,46]$ is not necessary because the formulation is naturally singularity-free. The present combination of update scheme with IGA-C has the additional advantages over the total Lagrangian Galerkinbased schemes of: (i) reflecting the theoretical kinematic model in a very simple and natural way, where only the familiar concept of linearization is required and no other geometric operators are involved; (ii) leading to simple linearized equations; (iii) requiring a minimal computational effort since in IGA-C only one point evaluation per element is required; (iv) avoiding the variational formulation. Moreover, it is expected in future studies that higher order approximation in the context of geometrically nonlinear problem will lead to improved results in the study of geometric instability and buckling phenomena [54-56].

Finally, we remark that to achieve the main objective of the paper, which is the extension of the IGA-C to geometrically exact beams, the paper presents some distinguishing features that are worth mentioning: linearization of higher-order derivatives of kinematic quantities due to the discretization of the strong form of the governing equations is provided; an original geometric interpretation of the beam is introduced; the classical kinematic assumption 
underlying the formulation of geometrically exact shear-deformable beams is given without introducing the concept of moving frame.

The outline of the paper is as follows. In Section 2 the kinematic formulation is presented. In Section 3 we briefly recall the governing equations, the stress-conjugate deformation measures and the constitutive equations. Section 4 addresses the key underlying geometric aspects necessary for the consistent linearization of the governing equations and for the consistent configuration update. Full linearization of the governing equations is given in Section 5 with the help of results given in Appendix A. In Section 6, we first briefly summarize the main facts regarding NURBS basis functions, then we collocate the linearized governing equations. Section 7, complemented with Appendix B, addresses the consistent update of all the kinematic variables. Numerical applications are presented in Section 8. Finally, in Section 9, a summary and the main conclusions of the work are drawn.

\section{Kinematics}

\subsection{Beam motion}

Let $\boldsymbol{E}$ be the affine environmental space and $\overline{\boldsymbol{E}}$ the associated vector space. We assume $\overline{\boldsymbol{E}}$ to be equipped with the standard Euclidean metric. Let $\boldsymbol{T}$ be the one-dimensional affine space of time instants.

The motion of a continuum body whose reference configuration is $\mathcal{B} \subset \boldsymbol{E}$ is the map

$$
\varphi: \boldsymbol{T} \times \mathcal{B} \rightarrow \boldsymbol{E}:(t, p) \mapsto \varphi(t, p) \in \boldsymbol{E} .
$$

We assume the motion $\varphi$ to be continuously differentiable up to the required order with respect to time and space. For a fixed time instant $t \in \boldsymbol{T}$, the motion $\varphi_{t}$ maps material points of $\mathcal{B}$ into the spatial domain $\boldsymbol{D}_{t}=\varphi_{t}(\mathcal{B}) \subset \boldsymbol{E}$. The map $\varphi_{t}$ is assumed to be regular.

Definition 2.1 (Definition of beam). We define beam to be the triple $(\mathcal{B}, \mathcal{S}, \pi)$, where

(i) $\mathcal{B}$ is a three-dimensional continuum body;

(ii) $\mathcal{S} \subset \mathcal{B}$ is a one-dimensional domain;

(iii) $\pi: \mathcal{B} \rightarrow \mathcal{S}$ is a surjective map (projection) such that for each $q \in \mathcal{S}, \mathcal{A}_{q}:=\pi^{-1}(q) \subset \boldsymbol{E}$ is a two-dimensional affine space called cross section of the beam;

(iv) The body $\mathcal{B}$ is the disjoint union of cross sections

$$
\mathcal{B}:=\bigcup_{q \in \mathcal{S}} \mathcal{A}_{q}
$$

(v) For each $q \in \mathcal{S}, q$ is the centroid of the cross section $\mathcal{A}_{q}$.

For each $q \in \mathcal{S}$, we choose the cross section $\mathcal{A}_{q}$ to be planar and orthogonal to the tangent vector to $\mathcal{S}$ at $q$. The boundary $\partial \mathcal{A}_{q}$ is assumed regular. In the following, we will refer to $\mathcal{S}$ as the centroid line of the beam.

From the topological point of view, in the definition given above, the beam is regarded to as a fibre bundle (see e.g. [42, p. 124]) where $\mathcal{S}$ is the base space and $\mathcal{A}_{q}$ the fibre at $q$. This geometric interpretation permits avoiding the concept of moving frame in the following kinematic formulation.

Assumption 2.1 (Fundamental kinematic assumption). The beam motion $\varphi: \boldsymbol{T} \times \mathcal{B} \rightarrow \boldsymbol{E}$ is given by

$$
\varphi(t, p)=\varphi(t, \pi(p))+\mathbf{R}(t, \pi(p))(p-\pi(p)) \text { for each } t \in \boldsymbol{T}, p \in \mathcal{B},
$$

where $\mathbf{R}: \boldsymbol{T} \times \mathcal{S} \rightarrow \mathrm{SO}(3)$.

The fundamental kinematic assumption represents a kinematic constraint to the general three-dimensional motion given in Eq. (1). It states that for each material point $p$ belonging to a beam cross section, the distance $\|p-q\|$ in the reference configuration, with $q=\pi(p)$, remains unchanged during the motion. Therefore, the fundamental kinematic assumption permits describing completely the motion of any material point $p$ of the beam through the motion of its projection $\pi(p)=q$ on the centroid line $\mathcal{S}$ "plus" the rotation $\mathbf{R}$ of the beam cross section $\mathcal{A}_{q}$ the material point $p$ belongs to. 
Roughly speaking, an affine space $\boldsymbol{E}$ consists in a set of points, an associated vector space $\overline{\boldsymbol{E}}$, and an operation, called translation map, through which a point of $\boldsymbol{E}$ is translated by an element of $\overline{\boldsymbol{E}}$ to another point of $\boldsymbol{E}$. We observe that in Eq. (2), the symbol + denotes the translation map of the affine space $\boldsymbol{E}$. Since the rotation operator $\mathbf{R}$ is a two-point tensor, which transforms vectors of the reference configuration into vectors of the current configuration, it makes sense to translate the point $\varphi(t, \pi(p))$ to $\varphi(t, p)$ through the vector $\mathbf{R}(t, \pi(p))(p-\pi(p))$ which is applied at $\varphi(t, \pi(p))$.

The fundamental kinematic assumption guarantees that the beam geometric structure given in Definition 2.1 is preserved by the motion. Cross sections in the reference configuration are transformed into cross sections in the current configuration, and material points belonging to the centroid line in the reference configuration are mapped into points belonging to the centroid line of the current configuration. Namely, for each $t \in \boldsymbol{T}$, the triple $\left(\boldsymbol{D}_{t}, \boldsymbol{S}_{t}, \pi_{t}\right)$ is a beam, where $\boldsymbol{S}_{t}=\varphi_{t}(\mathcal{S})$ and $\pi_{t}$ is the projection at time $t$.

We observe that Assumption 2.1 rephrases equation 1.6 of Ref. [34] with the difference that, by exploiting the affine structure of the environmental space and that the rotation operator is a two-point tensor, it avoids introducing the moving frame.

Let $c:=\varphi_{\left.\right|_{\mathcal{S}}}: \boldsymbol{T} \times \mathcal{S} \rightarrow \boldsymbol{E}$ be the motion restricted to the centroid curve $\mathcal{S}$, namely $c(t, q)=\varphi(t, \pi(p))$, with $q=\pi(p)$. The motion given in Eq. (2) can be equivalently written as

$$
\varphi(t, p)=c(t, q)+\mathbf{R}(t, q)(p-q) \quad \text { for each } t \in \boldsymbol{T}, q \in \mathcal{S} .
$$

For each $t \in \boldsymbol{T}$ and $p \in \mathcal{B}$, the deformation gradient of a beam is given by [34,46]

$$
\mathbf{F}(t, p)=\mathbf{n} \otimes\left[c,_{s}(t, q)+\tilde{\boldsymbol{\kappa}}(t, q)(\varphi(t, p)-c(t, q))\right]+\mathbf{R}(t, q)
$$

where

$$
\tilde{\boldsymbol{\kappa}}=\mathbf{R},{ }_{s} \mathbf{R}^{\top}: \boldsymbol{T} \times \mathcal{S} \rightarrow \operatorname{so}(3)
$$

is a skew-symmetric tensor field called spatial curvature of the beam and $\mathbf{n}$ is the unit vector orthogonal to the cross section $\mathcal{A}_{q}$.

In the above equations we have introduced the one-dimensional coordinate system $s: \mathcal{S} \rightarrow \mathcal{I}_{s}=[0, L] \subset \mathbb{R}$, where $L$ denotes the length of the centroid line in the reference configuration. $(\cdot),{ }_{s}$ indicates the standard derivative with respect to the coordinate $s$.

The beam axis curvature can be equivalently expressed by means of the material curvature tensor, which is a skew-symmetric tensor $\widetilde{\boldsymbol{K}}: \boldsymbol{T} \times \mathcal{S} \rightarrow$ so(3) defined as

$$
\widetilde{\boldsymbol{K}}(t, q)=\mathbf{R}^{\top} \mathbf{R},,_{s} .
$$

Spatial and material curvature tensors are related by

$$
\widetilde{\boldsymbol{K}}(t, q)=\mathbf{R}^{\top} \tilde{\boldsymbol{\kappa}}(t, q) \mathbf{R} \text { and } \tilde{\boldsymbol{\kappa}}(t, q)=\mathbf{R} \widetilde{\boldsymbol{K}}(t, q) \mathbf{R}^{\top} .
$$

For additional details on spatial and material objects one can refer to [40,46,49]. We observe that from the Lie groups standpoint, the spatial curvature tensor turns out to be $\tilde{\boldsymbol{\kappa}}=\operatorname{Ad}(\mathbf{R}) \widetilde{\boldsymbol{K}}(t, q)$, where Ad is the adjoint representation of $\mathrm{SO}(3)$ on so(3) [42, p. 167].

\section{One-dimensional balance equations and stress-conjugate strain measures}

In this section we briefly recall from [34] the one-dimensional governing equations for a beam in the static case.

\subsection{Balance equations}

By using the definitions of internal forces and couples given in [34], together with the balance equations for a three-dimensional continuum body (see for example [57,58]), the reduced form of translational and rotational equilibrium equations are obtained as follows

$$
\begin{aligned}
\boldsymbol{n},{ }_{s}+\boldsymbol{n}^{\mathrm{ext}} & =0 \\
\boldsymbol{m},{ }_{s}+c,{ }_{s} \times \boldsymbol{n}+\boldsymbol{m}^{\mathrm{ext}} & =0,
\end{aligned}
$$


where $\boldsymbol{n}, \boldsymbol{m}$ and $\boldsymbol{n}^{\text {ext }}$ and $\boldsymbol{m}^{\text {ext }}$ are the unit-length internal and external forces and moments, respectively. The complete derivation of the above equations from the three-dimensional form of the balance equations is given in [34].

\subsection{Deformation measures}

The stress-conjugate strain measures for a beam are derived by forcing the internal power of the beam regarded as a three-dimensional body to be equal to the internal power of the beam regarded as a one-dimensional reduced body. Namely,

$$
\int_{\mathcal{B}} \mathbf{P}(t, p): \dot{\mathbf{F}}(t, p) d V=\int_{\mathcal{S}} \boldsymbol{n}(t, q) \cdot \check{\dot{\gamma}}(t, q)+\boldsymbol{m}(t, q) \cdot \check{\dot{\boldsymbol{\kappa}}}(t, q) d S
$$

where $\mathbf{P}(t, p)$ is the first Piola-Kirchhoff stress tensor and $\dot{\mathbf{F}}(t, p)$ is the time derivative of the deformation gradient. $\check{\dot{\gamma}}(t, q)$ and $\check{\boldsymbol{\kappa}}(t, q)$ denote the objective rates of the stress-conjugate strain measures $\boldsymbol{\kappa}(t, q)$, defined as the axial vector of $\tilde{\boldsymbol{\kappa}}(t, q)$, and $\boldsymbol{\gamma}(t, q)$ defined as

$$
\boldsymbol{\gamma}(t, q)=c,{ }_{s}(t, q)-\mathbf{R}(t, q) \mathbf{n} .
$$

We refer to [34] for the proof of the above statements.

We recall that the axial vector of the skew-symmetric tensor $\tilde{\boldsymbol{\kappa}}(t, q)$ is a vector $\boldsymbol{\kappa}(t, q) \in \mathbb{R}^{3}$ such that $\boldsymbol{\kappa} \times \boldsymbol{h}=\tilde{\boldsymbol{\kappa}} \boldsymbol{h}$, for any $\boldsymbol{h} \in \mathbb{R}^{3}$. This can be generalized to any skew-symmetric tensor field.

The objective rate, also referred to as the Lie time derivative, is defined as $\stackrel{(\cdot)}{(\cdot)}:=\partial(\cdot) / \partial t-\widetilde{\boldsymbol{\omega}}(\cdot)$, where $\widetilde{\boldsymbol{\omega}}:=\dot{\mathbf{R}} \mathbf{R}^{\top}$ is the spatial angular velocity tensor. As pointed out in [34], from the physical viewpoint, the objective rate of the strain measures represents their rate relative to an observer moving with the beam cross section. Therefore, the effect of the cross section angular velocity is subtracted from the total rate. More in general, the Lie derivative is performed by first pulling-back the spatial quantity to the reference manifold, taking there the time derivative, and then pushing-forward the result to the current manifold (configuration). In our case, pull-back and push-forward operations are carried out through $\mathbf{R}^{\top}$ and $\mathbf{R}$, respectively. See [57,58] for a formal definition of Lie derivative and [40,52,50] for its systematic use in the framework of geometrically nonlinear beam formulation.

For our purposes it is more convenient to write the constitutive equations in the material form. To this aim, we pull-back to the reference configuration the spatial forces and the strain measures as follows

$$
\begin{array}{llll}
\boldsymbol{N}(t, q) & =\mathbf{R}(t, q)^{\top} \boldsymbol{n}(t, q) & \text { and } & \boldsymbol{M}(t, q)=\mathbf{R}(t, q)^{\top} \boldsymbol{m}(t, q), \\
\boldsymbol{\Gamma}(t, q)=\mathbf{R}(t, q)^{\top} \boldsymbol{\gamma}(t, q) & \text { and } & \boldsymbol{K}(t, q)=\mathbf{R}(t, q)^{\top} \boldsymbol{\kappa}(t, q),
\end{array}
$$

where $\boldsymbol{N}$ and $\boldsymbol{M}$ denote, respectively, the internal forces and couples in the material form, and $\boldsymbol{\Gamma}$ and $\boldsymbol{K}$ denote the material form of the strain measures. $\widetilde{\boldsymbol{K}}(t, q)$.

We observe that $\boldsymbol{K}(t, q)$ represents the axial vector of the associated skew-symmetric material curvature tensor

Invoking the orthogonal property of the rotation operator, we have that the axial and shear strain measures vector can be equivalently expressed as

$$
\boldsymbol{\Gamma}(t, q)=\mathbf{R}(t, q)^{\top}\left[c,{ }_{s}(t, q)-\mathbf{R}(t, q)\right]=\mathbf{R}(t, q)^{\top} c,_{s}(t, q)-\mathbf{n} .
$$

\subsection{Constitutive equations}

We restrict the present study to the case of Saint Venant-Kirchhoff materials.

The material internal forces and couples are linearly related to the material strain measures as follows [40,46,49]

$$
\boldsymbol{N}(t, q)=\mathbb{C}_{N} \boldsymbol{\Gamma}(t, q) \text { and } \boldsymbol{M}(t, q)=\mathbb{C}_{M} \boldsymbol{K}(t, q),
$$

where

$$
\mathbb{C}_{N}=\operatorname{diag}\left(G A_{1}, G A_{2}, E A\right) \quad \text { and } \quad \mathbb{C}_{M}=\operatorname{diag}\left(E J_{1}, E J_{2}, G J\right) .
$$

$G A_{1}$ and $G A_{2}$ are the shear stiffnesses along the cross section $\mathcal{A}_{q}$ principal axes, $E A$ is the axial stiffness. $G J$ is the torsional stiffness and $E J_{1}$ and $E J_{2}$ are the principal bending stiffnesses. 


\section{The configuration manifold and its tangent space}

In this section we recall the main geometric features of the underlying kinematic model that are fundamental for the subsequent numerical formulation.

With the fundamental kinematic assumption given in Eq. (2) (or (3)) in force, a spatial configuration of the beam is fully determined by the pair $(c, \mathbf{R})$, where $c$ is a map onto the affine Euclidean space $\boldsymbol{E}$ and $\mathbf{R}$ is a map onto the Special Orthogonal group $\mathrm{SO}(3)$.

For a beam, the set of all possible configurations is given by

$$
\mathcal{C}=\{(c, \mathbf{R}) \mid c: \boldsymbol{T} \times \mathcal{S} \rightarrow \boldsymbol{E}, \mathbf{R}: \boldsymbol{T} \times \mathcal{S} \rightarrow \mathrm{SO}(3)\} .
$$

In the language of differential geometry, $\mathcal{C}$ represents a differential manifold called configuration manifold.

The concept of (linear) approximation, which is the main idea behind the linearizations performed in the next section, consists in replacing locally a nonlinear manifold with its tangent space. This process generalizes the classical linearization procedure normally used in nonlinear continuum mechanics [59].

The tangent space to the configuration manifold at $(c, \mathbf{R}) \in \mathcal{C}$ is given by

$$
T_{(c, \mathbf{R})} \mathcal{C}=T_{c} \boldsymbol{E} \times T_{\mathbf{R}} \mathrm{SO}(3) .
$$

From the physical point of view, the tangent space is the vector space where the configuration increments live. In the weak formulation context, we note that the tangent space represents also the vector space of virtual displacements [60].

Let $\varepsilon \mapsto \gamma(\varepsilon)=\left(c_{\varepsilon}, \mathbf{R}_{\varepsilon}\right) \in \mathcal{C}$ be a curve on $\mathcal{C}$ such that $\gamma(0)=(c, \mathbf{R})$. By definition of tangent space, see for example [42, p. 120], $d \gamma(\varepsilon) /\left.d \varepsilon\right|_{\varepsilon=0}$ represents a tangent vector to $\mathcal{C}$ at $(c, \mathbf{R})$.

With the aid of the exponential map (e.g. [42, p. 160] and [43, p. 273]), we construct the curve $\gamma$ in a consistent way with respect to the configuration manifold as follows

$$
\gamma(\varepsilon)=\left(c_{\varepsilon}, \mathbf{R}_{\varepsilon}\right):=(c+\varepsilon \boldsymbol{\eta}, \exp (\varepsilon \widetilde{\vartheta}) \mathbf{R}),
$$

where $\boldsymbol{\eta} \in T_{c} \boldsymbol{E}$ and $\widetilde{\boldsymbol{\vartheta}} \in \operatorname{so}(3)$. The consistency with the underlying geometric structure of the configuration manifold $\mathcal{C}$ is guaranteed because $\left(c_{\varepsilon}, \mathbf{R}_{\varepsilon}\right) \in \mathcal{C}$. In fact, $c_{\varepsilon}$ is a map onto $\boldsymbol{E}$ and $\mathbf{R}_{\varepsilon}$ is a map onto $\mathrm{SO}$ (3). While the former can be understood intuitively thanks to the affine structure of $\boldsymbol{E}$, for the latter we observe that $\mathbf{R}_{\varepsilon}=\exp (\varepsilon \widetilde{\boldsymbol{\vartheta}}) \mathbf{R}$ represents the composition of two subsequent rotations whose result is still a rotation [38]. Formally, $\mathbf{R}_{\varepsilon}$ is called (left) translation and represents a transformation of the group into itself, $\mathrm{SO}(3) \times \mathrm{SO}(3) \rightarrow \mathrm{SO}(3)$. This formulation relies on the crucial fact that by definition exp maps a line $\varepsilon \widetilde{\vartheta}$ in the tangent space at the identity of SO(3), denoted by so(3), onto the group itself $\mathrm{SO}(3)$.

It follows that the tangent vector at $(c, \mathbf{R}) \in \mathcal{C}$ is given by

$$
\left.\frac{d}{d \varepsilon} \gamma(\varepsilon)\right|_{\varepsilon=0}=(\boldsymbol{\eta}, \widetilde{\vartheta} \mathbf{R})
$$

where $\boldsymbol{\eta} \in T_{c} \boldsymbol{E}$ and $\tilde{\vartheta} \mathbf{R} \in T_{\mathbf{R}} \operatorname{SO}(3)$.

We observe that the tangent space to the manifold $\boldsymbol{E}$ (the affine environmental space) at $c$ is the standard vector space $\overline{\boldsymbol{E}} \simeq \mathbb{R}^{3}$ applied in $c$. Whereas, the (spatial) tangent space to the $\operatorname{SO}(3)$ at $\mathbf{R}$ is defined as $T_{\mathbf{R}} \operatorname{SO}(3):=$ $\{\tilde{\boldsymbol{\vartheta}} \mathbf{R} \mid \tilde{\boldsymbol{\vartheta}} \in \operatorname{so}(3), \mathbf{R} \in \mathrm{SO}(3)\}[61,45]$.

From the physical point of view, $\boldsymbol{\eta}(t, q)$ represents an incremental displacement field superimposed to the current (spatial) configuration of the centroid line $c(t, q)$; whereas $\tilde{\vartheta}(t, q)$, such that $\tilde{\vartheta} \mathbf{R} \in T_{\mathbf{R}} \operatorname{SO}(3)$, represents an incremental rotation field superimposed to the current (spatial) rotation field $\mathbf{R}(t, q)$.

Note that we have chosen the spatial formulation (left translation) for the construction of the tangent space. An equivalent approach, leading to the material tangent space, can be used by employing a right translation of the current rotation $\mathbf{R}[46,44,49,45]$.

We now recall that given a skew-symmetric tensor $\widetilde{\psi} \in \operatorname{so}(3)$, the rotation vector $\boldsymbol{\psi}$ is the axial vector of $\widetilde{\psi}[62,38$, 51,43]. Physically, the rotation vector $\psi$ represents a rotation of an angle $\psi=\|\boldsymbol{\psi}\|$ around the rotation axis defined by the unit vector $\psi / \psi$. The closed-form expression relating the rotation vector $\psi$ to the rotation operator $\mathbf{R}$ is the 
Rodrigues formula given by

$$
\mathbf{R}=\exp (\widetilde{\boldsymbol{\psi}})=\operatorname{id}_{\mathrm{SO}(3)}+\frac{\sin (\psi)}{\psi} \tilde{\boldsymbol{\psi}}+\frac{1}{2}\left(\frac{\sin (\psi / 2)}{\psi / 2}\right)^{2} \widetilde{\boldsymbol{\psi}}^{2} .
$$

We emphasize that, in general, the rotation vector depends both on time and space (the centroid line $\mathcal{S}$ ), implying the instantaneous change of the rotation axis for each different beam cross section.

In our displacement-based formulation we adopt as independent kinematic variables the vector fields $\eta(t, q)$ and $\vartheta(t, q)$. The updating procedure discussed later in Section 7 crucially relies on the combination of Eq. (19) (where the generic tensor field $\widetilde{\psi} \in \operatorname{so}(3)$ is replaced by the incremental rotation $\widetilde{\vartheta}(t, q))$ and Eq. (17).

As opposed to total Lagrangian formulations based on total rotation vector parametrization (see for example $[46,48,49])$ we point out that in our formulation, since the incremental rotations are always small quantities, we never incur singularity problems.

\section{Consistent linearization of the governing equations}

The linearization of the governing equation is based on a systematic use of directional derivatives [59] that in the present case are adjusted in order to guarantee the consistency with the geometric structure of the underlying configuration manifold discussed in the previous section.

\subsection{Consistent linearization of the strong form}

For a displacement-based numerical formulation we need to express the governing equations in terms of kinematic quantities. We first transform the balance equations (6) and (7) in the material form by means of Eqs. (10), and then we make use of the constitutive equations (13). The material form of Eqs. (6) and (7) in terms of kinematic quantities is

$$
\begin{array}{r}
\widetilde{\boldsymbol{K}} \mathbb{C}_{N} \boldsymbol{\Gamma}+\mathbb{C}_{N} \boldsymbol{\Gamma},{ }_{s}+\mathbf{R}^{\top} \boldsymbol{n}^{\text {ext }}=0 \\
\widetilde{\boldsymbol{K}} \mathbb{C}_{M} \boldsymbol{K}+\mathbb{C}_{M} \boldsymbol{K},{ }_{s}+\mathbf{R}^{\top} c,{ }_{s} \times \mathbb{C}_{N} \boldsymbol{\Gamma}+\mathbf{R}^{\top} \boldsymbol{m}^{\text {ext }}=0 .
\end{array}
$$

The boundary conditions at the beam free end $s=L$ are

$$
\begin{aligned}
\mathbf{R} \mathbb{C}_{N} \boldsymbol{\Gamma} & =\boldsymbol{n}_{c}^{\mathrm{ext}} \\
\mathbf{R} \mathbb{C}_{M} \boldsymbol{K} & =\boldsymbol{m}_{c}^{\mathrm{ext}},
\end{aligned}
$$

where $\boldsymbol{n}_{c}^{\text {ext }}$ and $\boldsymbol{m}_{c}^{\text {ext }}$ are the external concentrated forces and couples applied to the beam end in the current configuration.

In the above equations, the symbol $\widetilde{(\cdot)}$ denotes the skew-symmetric operator (sometimes called hat map). Also note that we have omitted the argument $(t, q)$ for the vector and tensor fields involved in the equations.

Proposition 5.1. The linearized form of Eq. (20) is

$$
\begin{aligned}
& {\left[\hat{\tilde{\boldsymbol{K}}} \mathbb{C}_{N} \hat{\mathbf{R}}^{\top} \tilde{c},_{s}-\mathbb{C}_{N} \hat{\tilde{\boldsymbol{K}}} \hat{\mathbf{R}}^{\top} \tilde{c},_{s}+\mathbb{C}_{N} \hat{\mathbf{R}}^{\top} \tilde{c},_{s s}+\hat{\mathbf{R}}^{\top} \widetilde{\boldsymbol{n}}^{\mathrm{ext}}\right] \boldsymbol{\vartheta}+\left[\mathbb{C}_{N} \hat{\mathbf{R}}^{\top} \hat{c},_{s}-\hat{\mathbf{R}}^{\top}\left(\widehat{\hat{\mathbf{R}} \mathbb{C}_{N} \hat{\boldsymbol{\Gamma}}}\right)\right] \boldsymbol{\vartheta},{ }_{s}} \\
& +\left[\hat{\widetilde{\boldsymbol{K}}} \mathbb{C}_{N} \hat{\mathbf{R}}^{\top}-\mathbb{C}_{N} \hat{\tilde{\boldsymbol{K}}} \hat{\mathbf{R}}^{\top}\right] \boldsymbol{\eta},{ }_{s}+\left[\mathbb{C}_{N} \hat{\mathbf{R}}^{\top}\right] \boldsymbol{\eta},{ }_{s s}=\hat{\boldsymbol{T}}_{N},
\end{aligned}
$$

where

$$
\hat{\boldsymbol{T}}_{N}=-\left(\hat{\tilde{\boldsymbol{K}}} \mathbb{C}_{N} \hat{\boldsymbol{\Gamma}}+\mathbb{C}_{N} \hat{\boldsymbol{\Gamma}},_{s}+\hat{\mathbf{R}}^{\top} \boldsymbol{n}^{\mathrm{ext}}\right) .
$$

Proof. We proceed by linearizing separately the three terms in Eq. (20). 
Linearization of the first term in Eq. (20)

$$
\begin{aligned}
L\left[\widetilde{\boldsymbol{K}} \mathbb{C}_{N} \boldsymbol{\Gamma}\right] & =\hat{\widetilde{\boldsymbol{K}}} \mathbb{C}_{N} \hat{\boldsymbol{\Gamma}}+\frac{d}{d \varepsilon}\left(\widetilde{\boldsymbol{K}}_{\varepsilon} \mathbb{C}_{N} \boldsymbol{\Gamma}_{\varepsilon}\right)_{\varepsilon=0} \\
& =\hat{\widetilde{\boldsymbol{K}}} \mathbb{C}_{N} \hat{\boldsymbol{\Gamma}}+\hat{\mathbf{R}}^{\top} \widetilde{\boldsymbol{\vartheta}},{ }_{s} \hat{\mathbf{R}} \mathbb{C}_{N} \hat{\boldsymbol{\Gamma}}+\hat{\widetilde{\boldsymbol{K}}} \mathbb{C}_{N} \hat{\mathbf{R}}^{\top} \boldsymbol{\eta},{ }_{s}-\hat{\widetilde{\boldsymbol{K}}} \mathbb{C}_{N} \hat{\mathbf{R}}^{\top}\left(\boldsymbol{\vartheta} \times \hat{\boldsymbol{c}},{ }_{s}\right),
\end{aligned}
$$

where Eqs. (A.9) and (A.13) in Appendix A have been used.

Linearization of the second term in Eq. (20)

$$
\begin{aligned}
L\left[\mathbb{C}_{N} \boldsymbol{\Gamma}_{\varepsilon, s}\right] & =\mathbb{C}_{N} \hat{\boldsymbol{\Gamma}},_{s}+\frac{d}{d \varepsilon}\left(\mathbb{C}_{N} \boldsymbol{\Gamma}_{\varepsilon, s}\right)_{\varepsilon=0} \\
& =\mathbb{C}_{N} \hat{\boldsymbol{\Gamma}},_{s}+\mathbb{C}_{N} \hat{\widetilde{\boldsymbol{K}}} \hat{\mathbf{R}}^{\top} \tilde{\boldsymbol{\vartheta}} \hat{c},{ }_{s}-\mathbb{C}_{N} \hat{\mathbf{R}}^{\top} \tilde{\boldsymbol{\vartheta}},_{s} \hat{c},{ }_{s}-\mathbb{C}_{N} \hat{\widetilde{\boldsymbol{K}}} \hat{\mathbf{R}}^{\top} \boldsymbol{\eta},{ }_{s}-\mathbb{C}_{N} \hat{\mathbf{R}}^{\top} \tilde{\boldsymbol{\vartheta}} \hat{c},{ }_{s s}+\mathbb{C}_{N} \hat{\mathbf{R}}^{\top} \boldsymbol{\eta},{ }_{s s},
\end{aligned}
$$

where Eq. (A.15) in Appendix A has been used.

Linearization of the third term of Eq. (20)

$$
L\left[\mathbf{R}_{\varepsilon}^{\top} n^{\text {ext }}\right]=\hat{\mathbf{R}}^{\top} \boldsymbol{n}^{\text {ext }}-\hat{\mathbf{R}}^{\top} \widetilde{\vartheta} n^{\text {ext }},
$$

where Eq. (A.4) in Appendix A has been used.

After a few manipulations and rearranging terms we obtain the proposition given.

Proposition 5.2. The linearized form of Eq. (21) is

$$
\begin{aligned}
& \left.\left[\widehat{\left(\hat{\mathbf{R}}^{\top} \hat{c},{ }_{s}\right.}\right) \mathbb{C}_{N} \hat{\mathbf{R}}^{\top} \tilde{\widetilde{c},{ }_{s}}-\widetilde{\left(\mathbb{C}_{N} \hat{\boldsymbol{\Gamma}}\right)} \hat{\mathbf{R}}^{\top} \tilde{\widetilde{c}}{ }_{s}+\hat{\mathbf{R}}^{\top} \widetilde{\boldsymbol{m}}^{\mathrm{ext}}\right] \boldsymbol{\vartheta}+\left[\hat{\tilde{\boldsymbol{K}}} \mathbb{C}_{M} \hat{\mathbf{R}}^{\top}-\hat{\mathbf{R}}^{\top}\left(\widehat{\hat{\mathbf{R}} \mathbb{C}_{M} \hat{\boldsymbol{K}}}\right)-\mathbb{C}_{M} \hat{\mathbf{R}}^{\top} \widetilde{(\hat{\mathbf{R}} \hat{\boldsymbol{K}})}\right] \boldsymbol{\vartheta},{ }_{s} \\
& \left.\left.+\left[\mathbb{C}_{M} \hat{\mathbf{R}}^{\top}\right] \boldsymbol{\vartheta},,_{s s}+\left[\widetilde{\left(\hat{\mathbf{R}}^{\top} \hat{c}, s\right.}\right) \mathbb{C}_{N} \hat{\mathbf{R}}^{\top}-\widetilde{\left(\mathbb{C}_{N} \hat{\boldsymbol{\Gamma}}\right.}\right) \mathbf{R}^{\top}\right] \eta,_{s}=\hat{\boldsymbol{T}}_{M},
\end{aligned}
$$

where

$$
\hat{\boldsymbol{T}}_{M}=-\left(\hat{\tilde{\boldsymbol{K}}} \mathbb{C}_{M} \hat{\boldsymbol{K}}+\mathbb{C}_{M} \hat{\boldsymbol{K}},_{s}+\left(\widetilde{\hat{\mathbf{R}}^{\top} \hat{c},{ }_{s}}\right) \mathbb{C}_{N} \hat{\boldsymbol{\Gamma}}+\hat{\mathbf{R}}^{\top} \boldsymbol{m}^{\mathrm{ext}}\right) .
$$

Proof. We proceed as in the previous case by linearizing separately the four terms in Eq. (21).

Linearization of the first term of Eq. (21)

$$
\begin{aligned}
L\left[\widetilde{\boldsymbol{K}} \mathbb{C}_{M} \boldsymbol{K}\right] & =\hat{\widetilde{\boldsymbol{K}}} \mathbb{C}_{M} \hat{\boldsymbol{K}}+\frac{d}{d \varepsilon}\left(\widetilde{\boldsymbol{K}}_{\varepsilon} \mathbb{C}_{M} \boldsymbol{K}_{\varepsilon}\right)_{\varepsilon=0} \\
& =\hat{\widetilde{K}} \mathbb{C}_{M} \hat{\boldsymbol{K}}+\hat{\mathbf{R}}^{\top} \widetilde{\boldsymbol{\vartheta}}{ }_{s} \hat{\mathbf{R}} \mathbb{C}_{M} \hat{\boldsymbol{K}}+\hat{\widetilde{\boldsymbol{K}}} \mathbb{C}_{M} \hat{\mathbf{R}}^{\top} \boldsymbol{\vartheta},{ }_{s},
\end{aligned}
$$

where Eqs. (A.9) and (A.12) in Appendix A have been used.

Linearization of the second term of Eq. (21)

$$
\begin{aligned}
L\left[\mathbb{C}_{M} \boldsymbol{K},{ }_{s}\right] & =\mathbb{C}_{M} \hat{\boldsymbol{K}},_{s}+\frac{d}{d \varepsilon}\left(\mathbb{C}_{M} \boldsymbol{K}_{\varepsilon, s}\right)_{\varepsilon=0} \\
& =\mathbb{C}_{M} \hat{\boldsymbol{K}},_{s}+\mathbb{C}_{M} \hat{\mathbf{R}}^{\top} \widetilde{\vartheta}{ }_{s} \hat{\mathbf{R}} \hat{\boldsymbol{K}}+\mathbb{C}_{M} \hat{\mathbf{R}}^{\top} \boldsymbol{\vartheta},{ }_{s s},
\end{aligned}
$$

where Eq. (A.20) in Appendix A has been used.

Linearization of the third term of Eq. (21)

$$
\begin{aligned}
L\left[\mathbf{R}^{\top} c,{ }_{s} \times \mathbb{C}_{N} \boldsymbol{\Gamma}\right]= & \hat{\mathbf{R}}^{\top} \hat{c},_{s} \times \mathbb{C}_{N} \hat{\boldsymbol{\Gamma}}+\frac{d}{d \varepsilon}\left(\mathbf{R}_{\varepsilon}^{\top} c_{\varepsilon, s} \times \mathbb{C}_{N} \boldsymbol{\Gamma}_{\varepsilon}\right)_{\varepsilon=0} \\
= & \hat{\mathbf{R}}^{\top} \hat{\boldsymbol{c}},_{s} \times \mathbb{C}_{N} \hat{\boldsymbol{\Gamma}}-\hat{\mathbf{R}}^{\top} \widetilde{\vartheta} \hat{c},{ }_{s} \times \mathbb{C}_{N} \hat{\boldsymbol{\Gamma}}+\hat{\mathbf{R}}^{\top} \boldsymbol{\eta},{ }_{s} \times \mathbb{C}_{N} \hat{\boldsymbol{\Gamma}} \\
& +\hat{\mathbf{R}}^{\top} \hat{\boldsymbol{c}},_{s} \times \mathbb{C}_{N} \hat{\mathbf{R}}^{\top}\left(\boldsymbol{\eta},{ }_{s}-\boldsymbol{\vartheta} \times \hat{c},,_{s}\right),
\end{aligned}
$$

where use has been made of Eqs. (A.1), (A.4) and (A.13) given in Appendix A. 
Linearization of the fourth term of Eq. (21)

$$
L\left[\mathbf{R}_{\varepsilon}^{\top} \boldsymbol{m}^{\mathrm{ext}}\right]=\hat{\mathbf{R}}^{\top} \boldsymbol{m}^{\mathrm{ext}}-\hat{\mathbf{R}}^{\top} \widetilde{\vartheta} \boldsymbol{m}^{\mathrm{ext}},
$$

where use has been made of Eq. (A.4).

Proposition 5.3. The linearized form of boundary condition given in Eq. (22) is

$$
\left[\hat{\mathbf{R}} \mathbb{C}_{N} \hat{\mathbf{R}}^{\top} \hat{\widetilde{c}},_{s}-\left(\widehat{\hat{\mathbf{R}} \mathbb{C}_{N} \hat{\boldsymbol{\Gamma}}}\right)\right] \boldsymbol{\vartheta}+\left[\hat{\mathbf{R}} \mathbb{C}_{N} \hat{\mathbf{R}}^{\top}\right] \boldsymbol{\eta},{ }_{s}=\hat{\boldsymbol{T}}_{N}^{b c},
$$

where

$$
\hat{\boldsymbol{T}}_{N}^{b c}=-\hat{\mathbf{R}} \mathbb{C}_{N} \hat{\boldsymbol{\Gamma}}+\boldsymbol{n}_{c}^{\mathrm{ext}}
$$

Proof.

$$
\begin{aligned}
L\left[\mathbf{R} \mathbb{C}_{N} \boldsymbol{\Gamma}-\boldsymbol{n}_{c}^{\mathrm{ext}}\right] & =\hat{\mathbf{R}} \mathbb{C}_{N} \hat{\boldsymbol{\Gamma}}-\boldsymbol{n}_{c}^{\mathrm{ext}}+\frac{d}{d \varepsilon}\left(\mathbf{R}_{\varepsilon} \mathbb{C}_{N} \boldsymbol{\Gamma}_{\varepsilon}\right)_{\varepsilon=0} \\
& =\hat{\mathbf{R}} \mathbb{C}_{N} \hat{\boldsymbol{\Gamma}}-\boldsymbol{n}_{c}^{\mathrm{ext}}+\tilde{\vartheta} \hat{\mathbf{R}} \mathbb{C}_{N} \hat{\boldsymbol{\Gamma}}+\hat{\mathbf{R}} \mathbb{C}_{N} \hat{\mathbf{R}}^{\top}\left(\boldsymbol{\eta},{ }_{s}-\vartheta \times \hat{c},{ }_{s}\right)=0,
\end{aligned}
$$

where use has been made of Eqs. (A.3) and (A.13).

Proposition 5.4. The linearized form of boundary condition (23) is

$$
\left[-\left(\widehat{\hat{\mathbf{R}} \mathbb{C}_{M} \hat{\boldsymbol{K}}}\right)\right] \boldsymbol{\vartheta}+\left[\hat{\mathbf{R}} \mathbb{C}_{M} \hat{\mathbf{R}}^{\top}\right] \boldsymbol{\vartheta},_{s}=\hat{\boldsymbol{T}}_{M}^{b c},
$$

where

$$
\hat{\boldsymbol{T}}_{M}^{b c}=-\hat{\mathbf{R}} \mathbb{C}_{M} \hat{\boldsymbol{K}}+\boldsymbol{m}_{c}^{\mathrm{ext}} .
$$

Proof.

$$
\begin{aligned}
L\left[\mathbf{R} \mathbb{C}_{M} \boldsymbol{K}-\boldsymbol{m}_{c}^{\mathrm{ext}}\right] & =\hat{\mathbf{R}} \mathbb{C}_{M} \hat{\boldsymbol{K}}-\boldsymbol{m}_{c}^{\mathrm{ext}}+\frac{d}{d \varepsilon}\left(\mathbf{R}_{\varepsilon} \mathbb{C}_{M} \boldsymbol{K}_{\varepsilon}\right)_{\varepsilon=0} \\
& =\hat{\mathbf{R}} \mathbb{C}_{M} \hat{\boldsymbol{K}}-\boldsymbol{m}_{c}^{\mathrm{ext}}+\tilde{\vartheta} \hat{\mathbf{R}} \mathbb{C}_{M} \hat{\boldsymbol{K}}+\hat{\mathbf{R}} \mathbb{C}_{M} \hat{\mathbf{R}}^{\top} \boldsymbol{\vartheta},{ }_{s}=0,
\end{aligned}
$$

where use has been made of Eqs. (A.3) and (A.12).

In the above propositions, the symbol $(\hat{)})$ indicates that the quantity in the parenthesis is evaluated at the configuration around which the linearization takes place.

\section{Discretization of the governing equations}

The above linearized governing equations are solved by adopting the concept of isogeometric analysis, namely both the geometry and the unknown variables are approximated by Non-Uniform Rational B-Splines (NURBS). In this section we firstly recall shortly the main features of B-splines and NURBS, restricting the focus only to the key geometric properties relevant to our case. For an exhaustive exposition of the subject we refer to [63]. Secondly, we discretize and collocate the linearized differential equations. 


\subsection{B-spline}

Let $U=\left[u_{0}, \ldots, u_{m}\right]$ be a nondecreasing sequence of $m+1$ real numbers called knot vector. The $i$ th B-spline basis function of $p$-degree, denoted by $N_{i, p}(u)$, is defined by means of the recursive formula

$$
\begin{aligned}
& N_{i, 0}(u)= \begin{cases}1 & \text { if } u_{i} \leq u<u_{i+1} \\
0 & \text { otherwise }\end{cases} \\
& N_{i, p}(u)=\frac{u-u_{i}}{u_{i+p}-u_{i}} N_{i, p-1}(u)+\frac{u_{i+p+1}-u}{u_{i+p+1}-u_{i+1}} N_{i+1, p-1}(u) .
\end{aligned}
$$

Namely, for $p>0, N_{i, p}$ is a linear combinations of two ( $\left.p-1\right)$-degree basis functions. By definition, Eq. (42) can yield the quotient $0 / 0$, which is defined to be zero. The most relevant properties for our purposes of the B-spline basis functions are the followings:

- $N_{i, p}(u)=0$ if $u$ is out of the interval $\left[u_{i}, u_{i+p+1}\right)$ (local support property).

- Given a knot span $\left[u_{i}, u_{i+1}\right)$, at most $p+1$ basis functions, namely $N_{i-p, p}, \ldots, N_{i, p}$, are nonzero.

- At a knot, $N_{i, p}(u)$ is $C^{(p-k)}$, where $k$ is the knot multiplicity.

A knot vector $U$ is said open (or nonperiodic) if the first and last knots have multiplicity $p+1$. A knot vector $U$ is said uniform if all the interior knots are equally spaced. Given an open knot vector with $m+1$ knots, there are $n+1$ basis functions with $n=m-p-1$. For the remainder of this work we will always use open knot vectors.

\subsection{NURBS}

A NURBS basis function of degree $p$ is defined as

$$
R_{i, p}(u)=\frac{N_{i, p}(u) w_{i}}{\sum_{j=0}^{n} N_{j, p}(u) w_{j}},
$$

where $N_{i, p}$ is a B-spline of degree $p$ and $w_{j} \in \mathbb{R}$ with $j=0, \ldots, n$ are called weights. The properties listed above for B-spline basis functions are naturally extended to NURBS basis functions. We note also that if all the B-spline basis functions in Eq. (43) have the same weight, namely $w_{j}=a, a \neq 0$ for all $j$, then $R_{i, p}(u)=N_{i, p}(u)$.

\subsection{Isoparametric representation}

Let $\mathcal{I}_{u}=\left[u_{0}, u_{m}\right] \subset \mathbb{R}$ be the normalized one-dimensional domain (parametric interval) of the NURBS basis functions, we have

$$
\begin{gathered}
c(u)=\sum_{j=0}^{n} R_{j, p}(u) \check{\boldsymbol{P}}_{j} \quad \text { with } u \in \mathcal{I}_{u}, \\
\boldsymbol{\eta}(u)=\sum_{j=0}^{n} R_{j, p}(u) \check{\boldsymbol{\eta}}_{j} \quad \text { with } u \in \mathcal{I}_{u}, \\
\boldsymbol{\vartheta}(u)=\sum_{j=0}^{n} R_{j, p}(u) \check{\boldsymbol{\vartheta}}_{j} \quad \text { with } u \in \mathcal{I}_{u},
\end{gathered}
$$

where $\check{\boldsymbol{P}}_{j} \in \boldsymbol{E}$, is the $j$ th control point and $\check{\boldsymbol{\eta}}_{j} \in \mathbb{R}^{3}, \check{\boldsymbol{\vartheta}}_{j} \in \mathbb{R}^{3}$ are the $j$ th control variables. As pointed out in [28], the displacement field $\boldsymbol{\eta}(u)$ and the rotation field $\vartheta(u)$ can be discretized by independent (different degree) NURBS basis functions. In the present study we adopt for both kinematic fields the same NURBS basis functions.

In order to express variables defined on the physical parametric interval $\mathcal{I}_{s}=[0, L] \subset \mathbb{R}$, we make use of a regular map $\alpha: \mathcal{I}_{u} \rightarrow \mathcal{I}_{s}: u \mapsto \alpha(u):=L u=s$, with its inverse $\alpha^{-1}(s):=s / L=u$, such that the following diagram commutes 


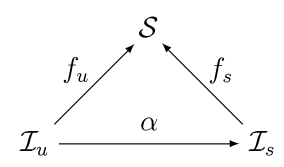

yielding

$$
f_{s}=f_{u} \circ \alpha^{-1},
$$

where $f_{u}$ denotes any of the three variables defined in the Eqs. (44)-(46) parametrized on $\mathcal{I}_{u}$.

The approximating NURBS basis functions defined on the physical space are given by $R_{j, p} \circ \alpha^{-1}$, with $j=$ $0, \ldots, n$. This composition has to be taken into account when derivatives with respect to the coordinate $s: \mathcal{S} \rightarrow \mathcal{I}_{s}$ are calculated. Namely,

$$
\begin{aligned}
& R_{j, p}^{\prime}:=\frac{d}{d s} R_{j, p}\left(\alpha^{-1}(s)\right)=\frac{1}{L} \frac{d}{d u} R_{j, p} \\
& R_{j, p}^{\prime \prime}:=\frac{d^{2}}{d s^{2}} R_{j, p}\left(\alpha^{-1}(s)\right)=\frac{1}{L^{2}} \frac{d^{2}}{d u^{2}} R_{j, p} .
\end{aligned}
$$

\subsection{Collocation and discretization of the governing equations}

Collocation can be either interpreted in a variational sense (e.g. [26,25,32,33]), where integrals in the weak form are eliminated by selecting the test function as the Dirac delta, formally constructed as the limit of a sequence of smooth functions with compact support that converge to a distribution, satisfying the so-called sifting property; or by enforcing directly the strong form of the governing equations to be satisfied in a discrete number of (collocation) points (e.g. $[23,27,28,24])$. We adopt the latter approach.

The linearized governing equations are collocated at the standard Greville abscissa [23]. The Greville abscissa related to a knot vector $U=\left[u_{0}, \ldots, u_{m}\right]$ of a $p$-degree $\mathrm{B}$-spline are points of the parametric space $\mathcal{I}_{u}$ given by

$$
u_{i}^{c}=\frac{u_{i+1}+\cdots+u_{i+p}}{p} \text { for } i=0, \ldots, n .
$$

Collocation can alternatively be made at the Greville abscissae associated with the second derivative spaces [26-28]. In this case the collocation points are less than the number of unknowns, since the knots used for computing them are smaller than the original knots. A square system matrix is recovered by adding the equations imposing the boundary conditions.

The $n-1$ collocated and discretized translational equations (see Eqs. (24) and (25)) are

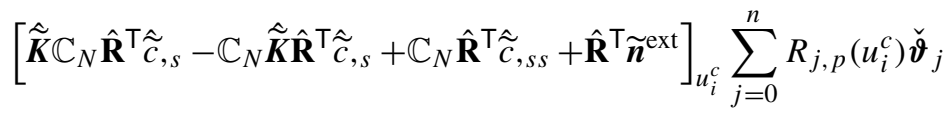

$$
\begin{aligned}
& +\left[\mathbb{C}_{N} \hat{\mathbf{R}}^{\top} \hat{\widetilde{c}}_{s}-\hat{\mathbf{R}}^{\top}\left(\widehat{\hat{\mathbf{R}} \mathbb{C}_{N} \hat{\boldsymbol{\Gamma}}}\right)\right]_{u_{i}^{c}} \sum_{j=0}^{n} R_{j, p}^{\prime}\left(u_{i}^{c}\right) \check{\vartheta}_{j} \\
& +\left[\hat{\widetilde{\boldsymbol{K}}} \mathbb{C}_{N} \hat{\mathbf{R}}^{\top}-\mathbb{C}_{N} \hat{\tilde{\boldsymbol{K}}} \hat{\mathbf{R}}^{\top}\right]_{u_{i}^{c}} \sum_{j=0}^{n} R_{j, p}^{\prime}\left(u_{i}^{c}\right) \check{\boldsymbol{\eta}}_{j}+\left[\mathbb{C}_{N} \hat{\mathbf{R}}^{\top}\right]_{u_{i}^{c}} \sum_{j=0}^{n} R_{j, p}^{\prime \prime}\left(u_{i}^{c}\right) \check{\boldsymbol{\eta}}_{j} \\
& =\hat{\boldsymbol{T}}_{N}\left(u_{i}^{c}\right), \quad \text { with } i=1, \ldots, n-1 .
\end{aligned}
$$

Eq. (51) represents a linear system of $(n-1)$ equations and $2(n+1)$ unknowns.

The $n-1$ collocated and discretized rotational equations (see Eqs. (29) and (30)) are

$$
\left.\left[\widetilde{\left(\hat{\mathbf{R}}^{\top} \hat{c},{ }_{s}\right.}\right) \mathbb{C}_{N} \hat{\mathbf{R}}^{\top} \tilde{\tilde{c},{ }_{s}}-\widetilde{\left(\mathbb{C}_{N} \hat{\boldsymbol{\Gamma}}\right)} \hat{\mathbf{R}}^{\mathrm{T}} \tilde{\tilde{c}}{ }_{s}+\hat{\mathbf{R}}^{\top} \widetilde{\boldsymbol{m}}^{\mathrm{ext}}\right]_{u_{i}^{c}} \sum_{j=0}^{n} R_{j, p}\left(u_{i}^{c}\right) \check{\boldsymbol{\vartheta}}_{j}
$$




$$
\begin{aligned}
& +\left[\hat{\tilde{\boldsymbol{K}}} \mathbb{C}_{M} \hat{\mathbf{R}}^{\top}-\hat{\mathbf{R}}^{\top}\left(\widehat{\left.\hat{\mathbf{R}} \mathbb{C}_{M} \hat{\boldsymbol{K}}\right)}-\mathbb{C}_{M} \hat{\mathbf{R}}^{\top} \widetilde{(\hat{\mathbf{R}} \hat{\boldsymbol{K}})}\right]_{u_{i}^{c}} \sum_{j=0}^{n} R_{j, p}^{\prime}\left(u_{i}^{c}\right) \check{\vartheta}_{j}\right.
\end{aligned}
$$

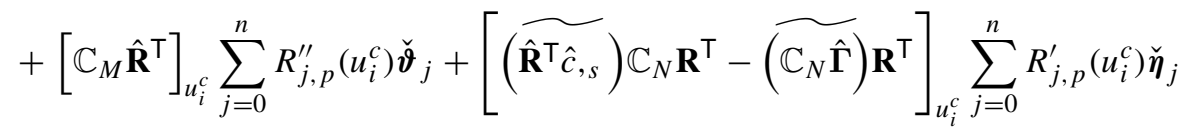

$$
\begin{aligned}
& =\hat{\boldsymbol{T}}_{M}\left(u_{i}^{c}\right), \quad \text { with } i=1, \ldots, n-1 .
\end{aligned}
$$

Eqs. (51) and (52) form a linear system of $2(n-1)$ equations with $2(n+1)$ unknowns. The four missing equations are provided by the boundary conditions.

By assuming a clamped end, the two discretized equations at the boundary $u_{0}^{c}$ are

$$
\sum_{j=0}^{n} R_{j, p}\left(u_{0}^{c}\right) \check{\vartheta}_{j}=0 \quad \text { and } \quad \sum_{j=0}^{n} R_{j, p}\left(u_{0}^{c}\right) \check{\eta}_{j}=0 .
$$

The translational discretized equation (see Eq. (35)) at the boundary $u_{n}^{c}$ is

$$
\left[\hat{\mathbf{R}} \mathbb{C}_{N} \hat{\mathbf{R}}^{\top} \hat{\tilde{c}},_{s}-\left(\widehat{\hat{\mathbf{R}} \mathbb{C}_{N} \hat{\boldsymbol{\Gamma}}}\right)\right]_{u_{i}^{c}} \sum_{j=0}^{n} R_{j, p}\left(u_{n}^{c}\right) \check{\vartheta}_{j}+\left[\hat{\mathbf{R}} \mathbb{C}_{N} \hat{\mathbf{R}}^{\top}\right]_{u_{i}^{c}} \sum_{j=0}^{n} R_{j, p}^{\prime}\left(u_{i}^{c}\right) \check{\boldsymbol{\eta}}_{j}=\hat{\boldsymbol{T}}_{N}^{b c} .
$$

The rotational discretized equation (see Eq. (38)) at the boundary $u_{n}^{c}$ is

$$
\left[-\left(\widehat{\hat{\mathbf{R}} \mathbb{C}_{M} \hat{\boldsymbol{K}}}\right)\right]_{u_{i}^{c}} \sum_{j=0}^{n} R_{j, p}\left(u_{n}^{c}\right) \check{\vartheta}_{j}+\left[\hat{\mathbf{R}} \mathbb{C}_{M} \hat{\mathbf{R}}^{\top}\right]_{u_{i}^{c}} \sum_{j=0}^{n} R_{j, p}^{\prime}\left(u_{n}^{c}\right) \check{\vartheta}_{j}=\hat{\boldsymbol{T}}_{M}^{b c} .
$$

The symbol $(\cdot)^{\prime}=d(\cdot) / d s$ and $(\cdot)^{\prime \prime}=d^{2}(\cdot) / d s^{2}$.

Eqs. (51) and (52) together with (53)-(55) form a linear system of $2(n+1)$ equations and $2(n+1)$ unknowns that can be rearranged in a standard form and solved by inverting the associated system matrix.

\section{Consistent incremental procedure and system matrix update}

Eqs. (24) and (29), with associated boundary conditions, represent only an approximate solution of the real nonlinear problem. They are valid only locally in the neighborhood of an equilibrium configuration; thus, the solution of the original nonlinear problem (Eqs. (20) and (21)) is found by solving multiple linear subproblems within a classical incremental-iterative procedure based on the Newton-Raphson algorithm.

Assume the configuration is known at the current $k$ th iteration. Namely, the set of control points $\check{\boldsymbol{P}}_{j}^{(k)}, j=0, \ldots, n$ and the rotation operators at the collocation points $\mathbf{R}^{(k)}\left(u_{i}^{c}\right), i=0, \ldots, n$ are known. The configuration update, as discussed in Section 4, is carried out through the control variables as follows.

Let $\check{\boldsymbol{\eta}}_{j}^{(k)}$ and $\check{\vartheta}_{j}^{(k)}$ be the solutions of the system matrix computed at the $k$ th iteration. Control points are updated by exploiting the standard translation operation in $\boldsymbol{E}$

$$
\check{\boldsymbol{P}}_{j}^{(k+1)}=\check{\boldsymbol{P}}_{j}^{(k)}+\check{\boldsymbol{\eta}}_{j}^{(k)} \quad \text { with } j=0, \ldots, n,
$$

from which we straightforwardly update the spatial configuration of the centroid line

$$
c^{(k+1)}\left(u_{i}^{c}\right)=\sum_{j=0}^{n} R_{j, p}\left(u_{i}^{c}\right) \check{\boldsymbol{P}}_{j}^{(k+1)} \quad \text { with } i=0, \ldots, n .
$$

For the rotation variables, we compute the incremental rotation vector and its derivatives at the collocation points

$$
\vartheta^{(k)}\left(u_{i}^{c}\right)=\sum_{j=0}^{n} R_{j, p}\left(u_{i}^{c}\right) \check{\vartheta}_{j}^{(k)} \quad \text { for } i=0, \ldots, n
$$




$$
\begin{aligned}
& \vartheta,,_{s}^{(k)}\left(u_{i}^{c}\right)=\sum_{j=0}^{n} R_{j, p}^{\prime}\left(u_{i}^{c}\right) \check{\vartheta}_{j}^{(k)} \quad \text { for } i=0, \ldots, n \\
& \boldsymbol{\vartheta},{ }_{s s}^{(k)}\left(u_{i}^{c}\right)=\sum_{j=0}^{n} R_{j, p}^{\prime \prime}\left(u_{i}^{c}\right) \check{\vartheta}_{j}^{(k)} \quad \text { for } i=0, \ldots, n .
\end{aligned}
$$

At the $i$ th collocation point, the rotation operator is then consistently updated as follows

$$
\mathbf{R}^{(k+1)}\left(u_{i}^{c}\right)=\exp \left(\vartheta^{(k)}\left(u_{i}^{c}\right)\right) \mathbf{R}^{(k)}\left(u_{i}^{c}\right) \quad \text { with } i=0, \ldots, n .
$$

To evaluate the updated system matrix at iteration $(k+1)$ (see $(\hat{.)}$ terms in Eqs. (51), (52), (54), and (55)) it is necessary to consistently update all the involved secondary kinematic quantities.

Update of the centroid line derivatives. First and second derivatives of the updated spatial configuration of the centroid line at the $(k+1)$ th iteration are given by

$$
c,_{s}^{(k+1)}=\sum_{j=0}^{n} R_{j, p}^{\prime} \check{\boldsymbol{P}}_{j}^{(k+1)} \quad \text { and } \quad c,{ }_{s s}^{(k+1)}=\sum_{j=0}^{n} R_{j, p}^{\prime \prime} \check{\boldsymbol{P}}_{j}^{(k+1)} .
$$

Update of the curvature tensor. At the $(k+1)$ th iteration, the material curvature tensor is given by

$$
\widetilde{\boldsymbol{K}}^{(k+1)}=\widetilde{\boldsymbol{K}}^{(k)}+\mathbf{R}^{\boldsymbol{\top}^{(k)}}\left(d \exp _{\widetilde{\vartheta}} \widetilde{\vartheta},_{s}^{(k)}\right) \mathbf{R}^{(k)} .
$$

The proof of the above proposition and some additional details on how to compute the derivative of the exponential $\operatorname{map} d \exp _{\widetilde{\vartheta}} \widetilde{\vartheta}{ }_{s}^{(k)}$ are given in Appendix B.

Update of the curvature tensor derivative. At the $(k+1)$ th iteration, the derivative of the material curvature tensor is given by

$$
\begin{aligned}
& \widetilde{\boldsymbol{K}},{ }_{s}^{(k+1)}=\widetilde{\boldsymbol{K}},{ }_{s}^{(k)}-\widetilde{\boldsymbol{K}}^{(k)} \mathbf{R}^{\boldsymbol{T}^{(k)}}\left(d \exp \widetilde{\boldsymbol{\vartheta}} \widetilde{\boldsymbol{\vartheta}},{ }_{s}^{(k)}\right) \mathbf{R}^{(k)} \\
& +\mathbf{R}^{\boldsymbol{T}^{(k)}}\left(d \exp _{\widetilde{\vartheta}} \widetilde{\boldsymbol{\vartheta}},_{s}^{(k)}\right) \mathbf{R}^{(k)} \widetilde{\boldsymbol{K}}^{(k)}+\mathbf{R}^{\boldsymbol{T}^{(k)}}\left(d \exp _{\widetilde{\vartheta}} \widetilde{\vartheta},{ }_{s}^{(k)}\right),_{s} \mathbf{R}^{(k)} .
\end{aligned}
$$

Comments on the above formula are given in Appendix B.

Update of the shear and axial strains measure vector. We make a direct use of the updated kinematic variables already presented

$$
\boldsymbol{\Gamma}^{(k+1)}=\mathbf{R}^{\boldsymbol{\top}^{(k+1)}} c,{ }_{s}^{(k+1)}-\mathbf{n} .
$$

Update of the shear and axial strains measure vector derivative. By making use of the updated curvature vector, we have

$$
\boldsymbol{\Gamma},{ }_{s}^{(k+1)}=-\widetilde{\boldsymbol{K}}^{(k+1)} \mathbf{R}^{\mathbf{T}^{(k+1)}} c,{ }_{s}^{(k+1)}+\mathbf{R}^{\mathbf{T}^{(k+1)}} c,{ }_{s s}^{(k+1)} .
$$

\section{Numerical results}

We present a series of numerical applications aimed at testing the capabilities of the proposed formulation. All tests are performed assuming maximum inter-element regularity (i.e. no internal knot repetitions, see third B-spline key property discussed in Section 6.1). Moreover, in all cases we employ the same degree for the B-spline basis functions for both translational and rotational kinematic variables.

\subsection{Roll-up of a beam under a concentrated end moment}

We first examine one of the most recurrent test case in the literature. It consists of a cantilever initially straight beam of length $L=10$ (placed along the $x_{2}$-axis) with a concentrated moment $\boldsymbol{m}=\boldsymbol{m} \boldsymbol{e}_{1}$ applied at its free end. 


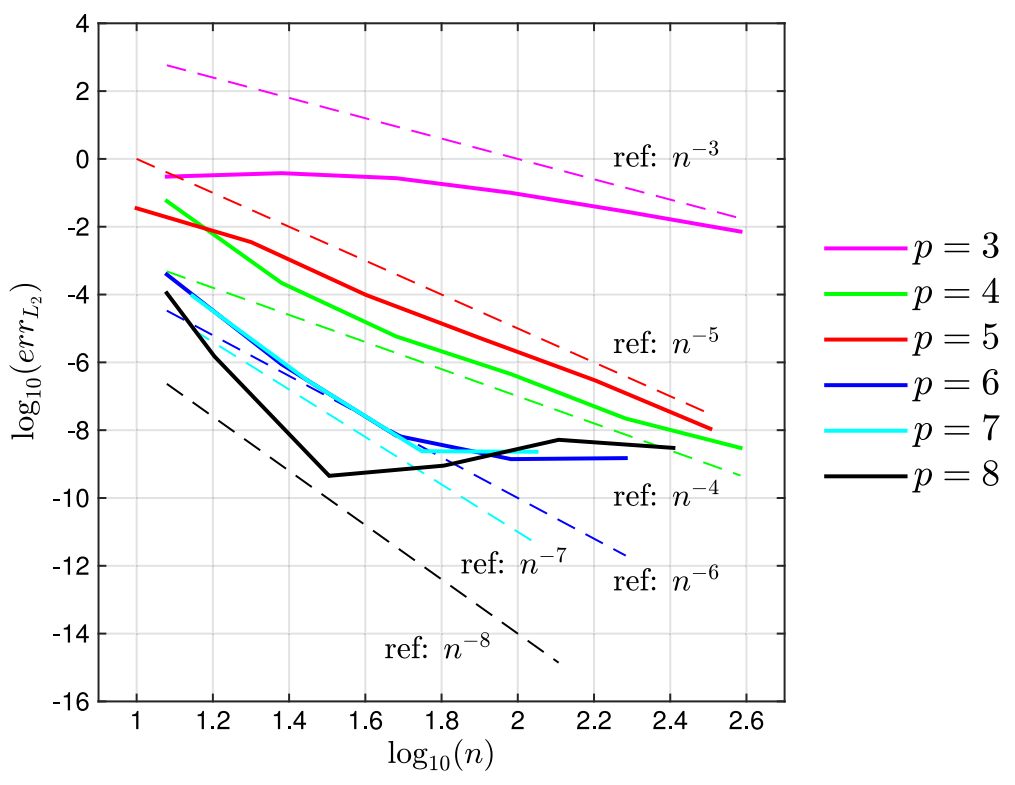

Fig. 1. Half-circle roll-up test of a cantilever beam with a concentrated couple at the free end. Spatial convergence plots for $p=3, \ldots, 8$ of the $L_{2}$-norm of the relative error as a function of the number of collocation points. Dashed lines: reference convergence rates.

We assign the same material properties as in [47]. The elasticity tensors are $\mathbb{C}_{N}=\operatorname{diag}\left(5 \times 10^{3}, 5 \times 10^{3}, 10^{4}\right)$ and $\mathbb{C}_{M}=\operatorname{diag}\left(10^{2}, 10^{2}, 10^{4}\right)$, respectively, which correspond to a beam with circular cross section with radius 0.2 and Young's modulus $E=79577$ and zero Poisson's ratio.

The beam is subjected to pure bending and its deformation occurs entirely in the plane $\left(x_{2}, x_{3}\right)$. The curvature of the beam is constant and equal to $m / E J_{1}$; the exact solution for the beam deformed axis is a circle with radius $E J_{1} / m$. An applied moment $m_{c}=2 \pi E J_{1} / L$ would force the beam to deform into a full closed circle with radius $L / 2 \pi$.

First test. In this test we assess the spatial convergence behavior of the proposed method. The test consists in applying a moment $m=m_{c} / 2$ in order to obtain a perfect semicircle with radius $L / \pi$. We compare the exact value of the free end position $\boldsymbol{P}^{e}$ with the one computed numerically $\boldsymbol{P}^{h}$. The $L_{2}$ norm of the error is calculated by $\operatorname{err}_{L_{2}}=\left\|\boldsymbol{P}^{e}-\boldsymbol{P}^{h}\right\|_{L_{2}} /\left\|\boldsymbol{P}^{e}\right\|_{L_{2}}$. Convergence curves associated with different basis function degrees $p$ are shown in Fig. 1. Reference rates of convergence (dashed lines) are added to the plot for comparison.

We observe that, excepting for $p=3$, for all degrees spatial convergence is obtained. Accuracy levels higher than $10^{-8}$ cannot be reached due to the stopping criterion used in the Newton-Raphson algorithm, where a tolerance of $10^{-8}$ for the error norm has been fixed. For this reason, in Fig. 1 relative error values of the convergence curves below this threshold are meaningless. Excepting for $p=5$, faster rates of convergence compared to the reference rates are observed for low numbers of collocation points. This appears to be more accentuated as the basis functions degree increases.

The poor accuracy convergence behavior for $p=3$ is ascribed to the higher order derivatives involved in the strong form of the governing equations. Second order derivatives of kinematic quantities like the curvature tensor imply linear approximating basis functions (see $R_{j, p}^{\prime \prime}$ in the above formulations), which turn out to be inappropriate. We remark that poor behavior for $p=3$ has been observed in nearly all works on IGA-C.

Some examples of deformed beam at initial, intermediate and final configuration for different basis function degrees $p$ and number of control points $n$ are shown in Fig. 2.

Second test. In the second numerical experiment regarding the beam roll-up we stress the proposed method in reproducing rotations larger than $2 \pi$ and again we evaluate the quality of the spatial convergence rate. We apply an end moment $m=2 m_{c}$, which forces the beam to wind around itself twice reaching a final circle of radius $L / 4 \pi$. The spatial position of the end point of the beam after the double roll-up coincides with the clamped end, being the latter located in the origin of the reference system. We evaluate the absolute error as $\operatorname{err}_{L_{2}}=\left\|\boldsymbol{P}^{h}\right\|_{L_{2}}$.

Spatial convergence plots are shown in Fig. 3. Also in this case, for all basis function degrees a satisfactory convergence rate is observed. Odd basis function degrees seem to have the same convergence rate of the lower even 

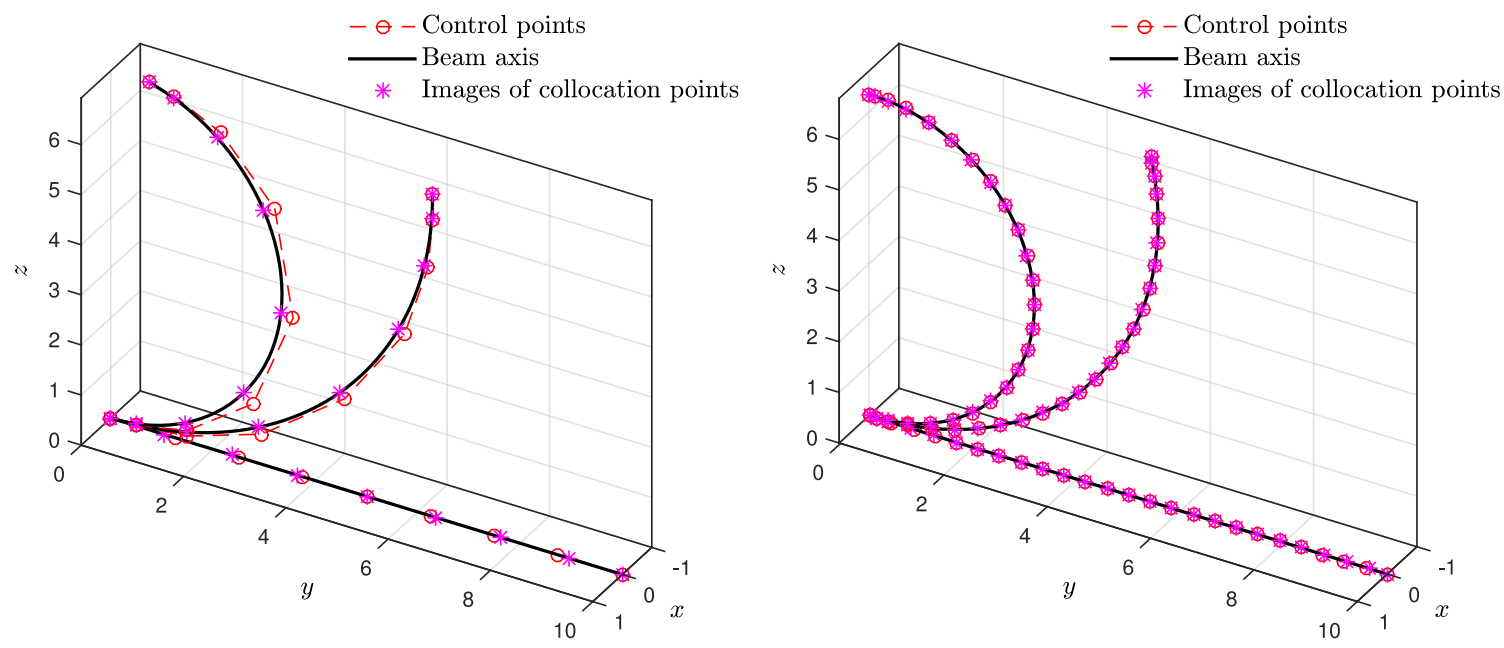

(a) $p=4, n=8$.

(b) $p=4, n=24$.
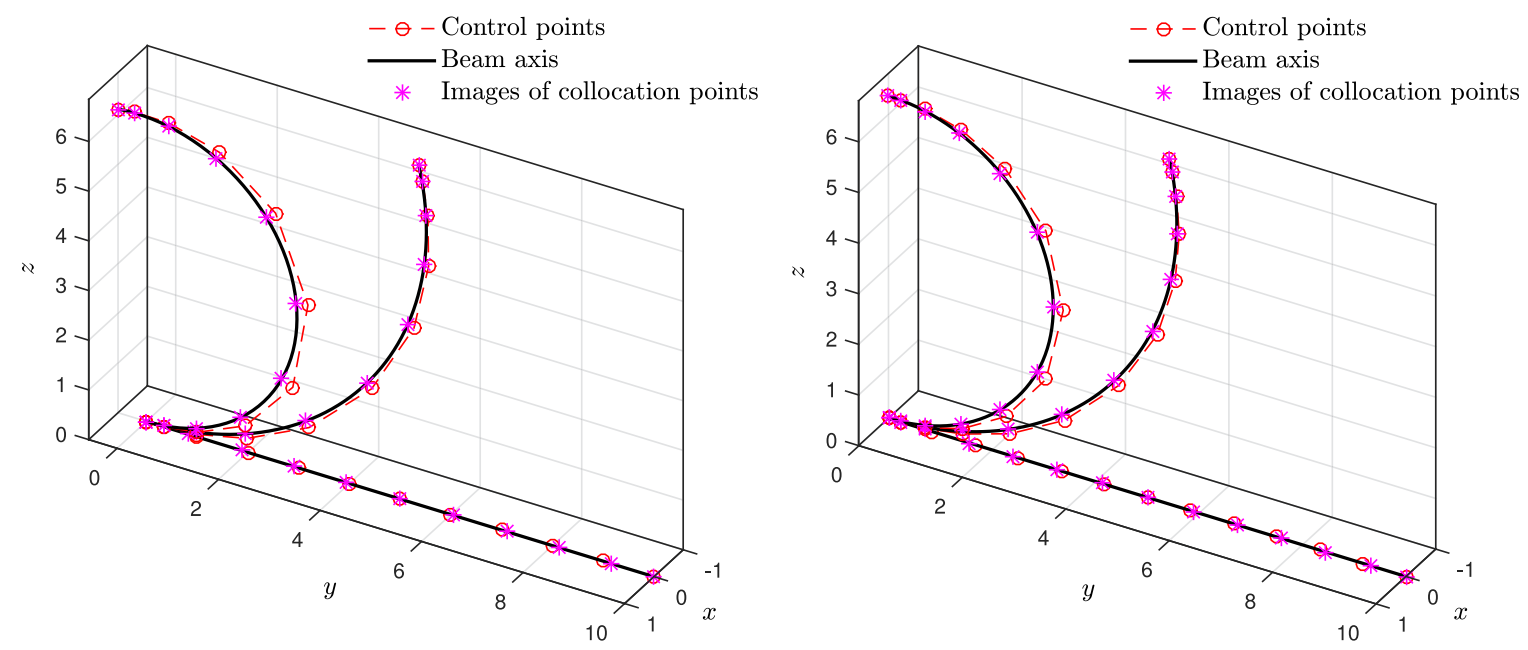

(c) $p=5, n=10$.

(d) $p=6, n=12$.

Fig. 2. Half-circle test: some examples of deformed beam at initial, intermediate and final configuration for different basis function degrees $p$ and number of control points $n$.

degree. The rate for $p=5$ is similar to $p=4$ and both are parallel to $n^{-4}$. A similar behavior is observed for $p=7$ and $p=6$ for $n>10^{1.8}$. As noticed in the previous case, excepting for $p=5$, faster rates of convergence compared to the reference ones are observed for lower numbers of collocation points. This appears to be more accentuated as the basis functions degree increases. Also in this case, error values below $10^{-8}$ are meaningless due to the stopping criterion of the Newton-Raphson algorithm.

Some examples of deformed beam at initial, one fourth, one half and final configuration for different basis function degrees $p$ and number of control points $n$ are shown in Figs. 4 and 5. With $p=4$ and $n=18$ the solution is poorly accurate (see Figs. 4(a) and 5(a)) where the two circles obtained when the full load is applied should be perfectly coincident. A much more accurate solution is obtained either by $n$ refinement (see Figs. 4(b) and 4(c)) or by order elevation (see Fig. 4(d)). Note that in the Fig. 4 the control polygon is not shown and images of collocation points are shown only at one fourth of the final load.

Fig. 5 shows the effects of $p$ and $n$ refinement for the double-circle test. We observe that since we are using NURBS basis function with all the same weights, that means B-spline basis functions, an exact circle cannot be 


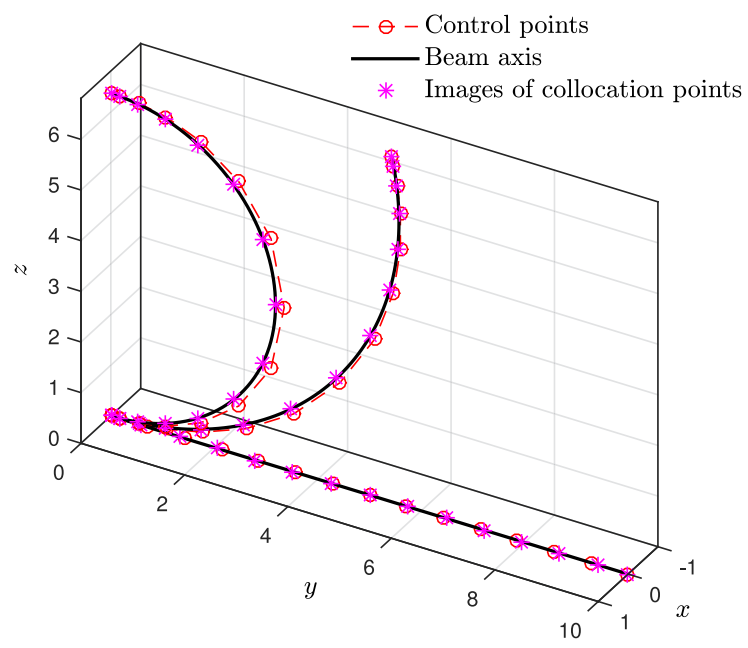

(e) $p=7, n=14$.

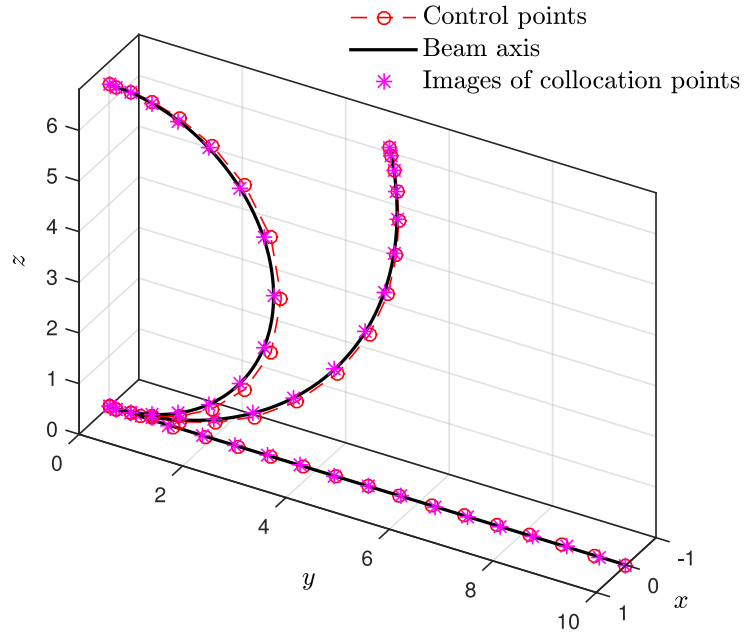

(f) $p=8, n=16$.

Fig. 2. (continued)

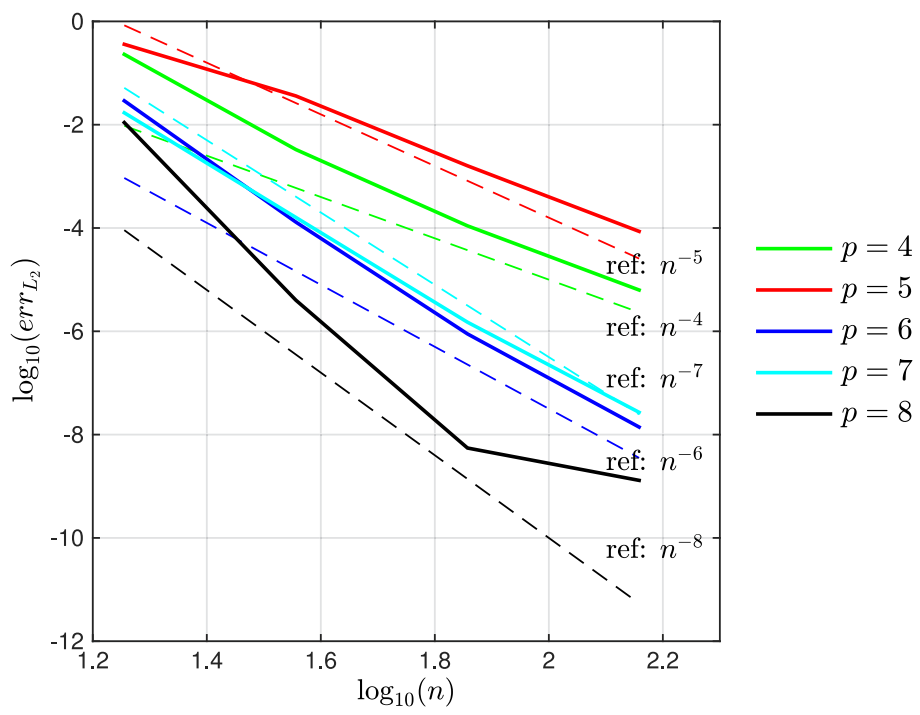

Fig. 3. Double-circle roll-up test of a cantilever beam with a concentrated couple at the free end. Spatial convergence plots for $p=4, \ldots, 8$ of the $L_{2}$-norm of the absolute error as a function of the number of collocation points. Dashed lines: reference convergence rates.

reproduced [63]. Nevertheless, the accuracy levels reached for example with $p=4$ and $n=72$ (Fig. 5(b)) and with $p=7$ and $n=36$ (Fig. 5(d)) are considered satisfactory.

\subsection{Helical beam test}

We repeat here the test first proposed in [50] that consists in bending an initially straight cantilever beam to an helical shape by applying simultaneously a concentrated couple $\boldsymbol{m}=m \boldsymbol{e}_{1}$ a and an out-of-plane concentrated force $\boldsymbol{f}=f \boldsymbol{e}_{1}$. The elasticity tensors are $\mathbb{C}_{N}=\operatorname{diag}\left(10^{4}, 10^{4}, 10^{4}\right)$ and $\mathbb{C}_{M}=\operatorname{diag}\left(10^{2}, 10^{2}, 10^{2}\right)$. This example is aimed at testing the capability of the present model when stressing three-dimensional conditions in terms of deformations are encountered. We assign $m=200 \pi$ and $f=50$ so that the beam is forced to wind around itself ten times with a rather complex three-dimensional deformation caused by the out-of-plane force. The initial configuration and the deformed shapes at $5 \%$ and $10 \%$ of the final loads are shown in Fig. 6. Fig. 7 shows the configurations of the deformed 

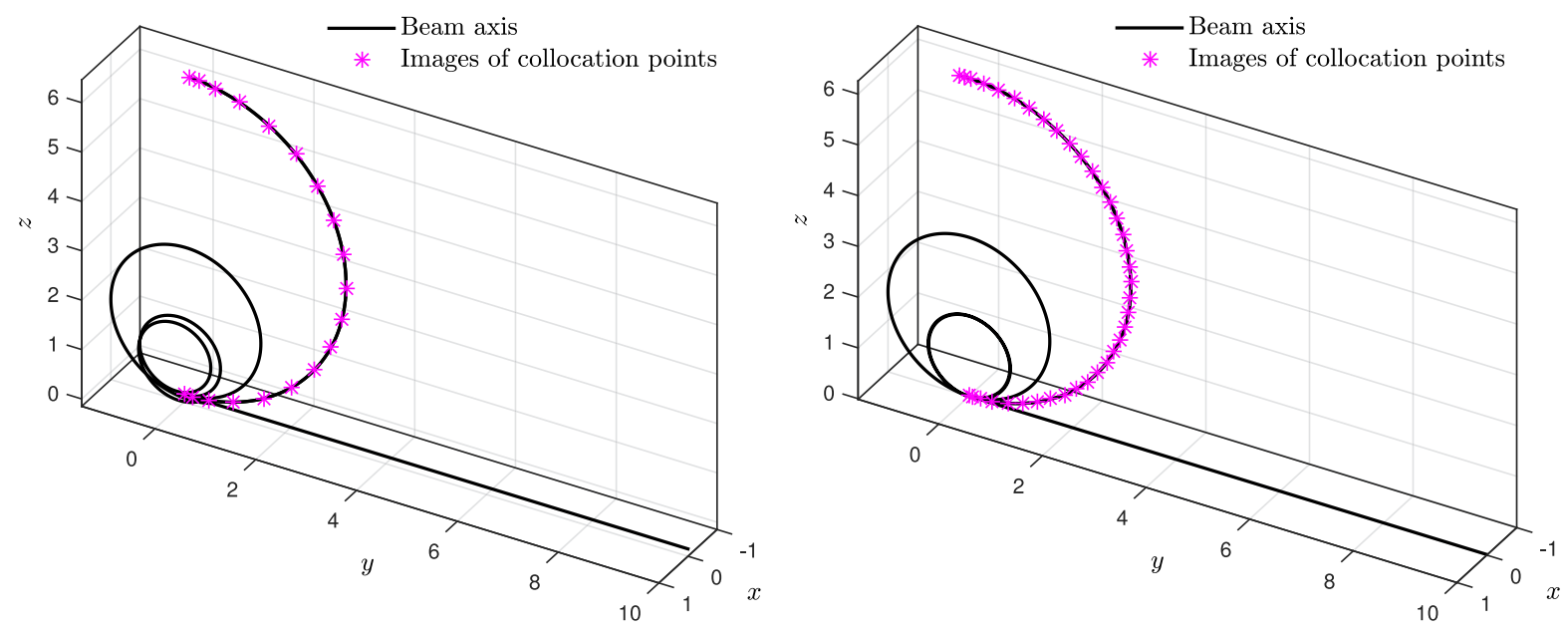

(a) $p=4, n=18$

(b) $p=4, n=36$.
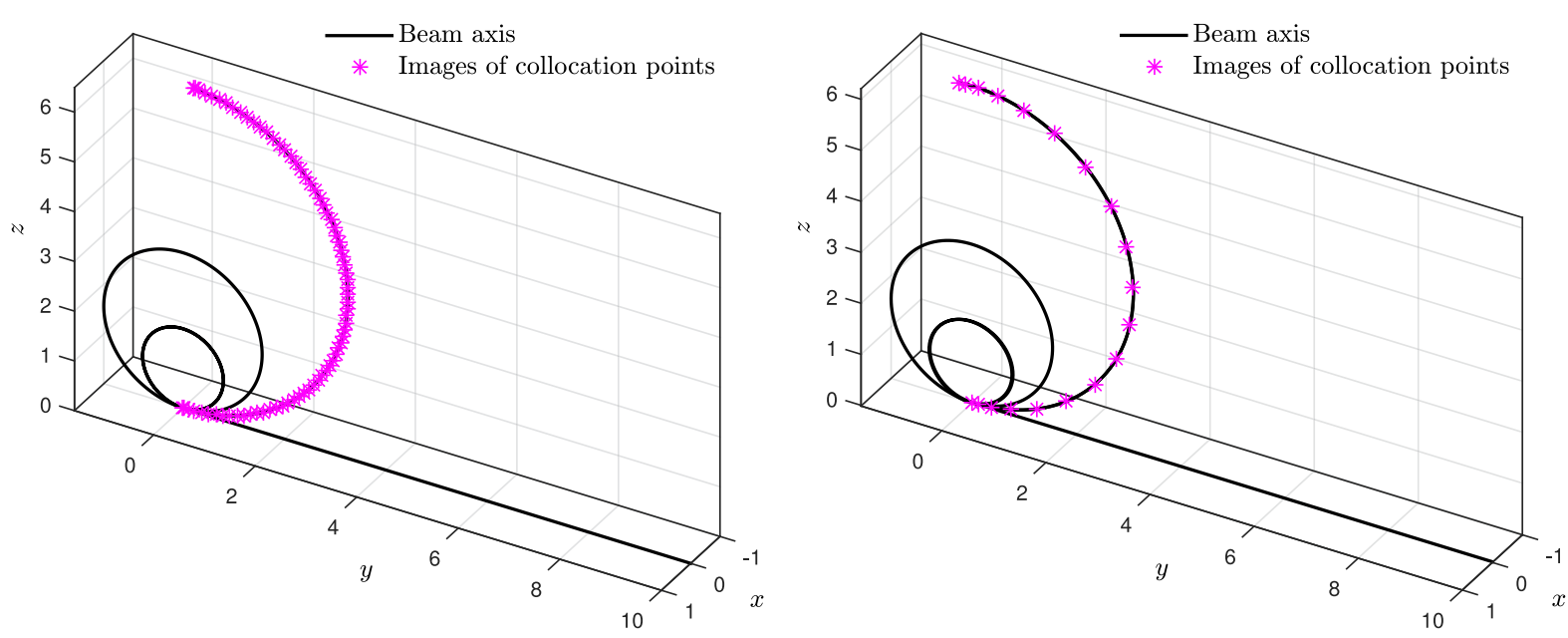

(c) $p=4, n=72$.

(d) $p=6, n=18$.

Fig. 4. Double-circle test: some examples of deformed beam at initial, one fourth, one and final configuration for different basis function degrees $p$ and number of control points $n$. Images of the collocation points are shown only when one fourth of the full load is applied.

beam for load levels from $15 \%$ to $65 \%$ of the final loads with increments of $10 \%$. Fig. 8 shows the configurations of the deformed beam for load levels from $75 \%$ to the full loads.

As the loads increase the free-end rotation keeps increasing, whereas the out-of-plane displacement takes oscillatory values around zero. In Fig. 9 we compare the out-of-plane displacement (in the $x_{1}$ direction) obtained with our model with results presented in [50,49,53] obtained with numerical schemes based on the FE formulation employing different rotations update concepts. In all the reported literature cases 2-node beam elements with linear shape functions are used. We observe excellent agreement with [50] (where 100 elements are used), especially up to $35 \%$ of the load. For larger loads we find a slightly better agreement with [49] (where 200 elements are used). The maximum out-of-plane displacement that occurs approximatively at 3.6\% of the full load (see Fig. 6) seems to be slightly overestimated by [49]. Results obtained by [53] show bigger differences with respect to [50,49] and to our results. ${ }^{1}$ Results provided by Mäkinen [49] with 100 elements (not shown here) based on the total rotation vector parametrization appear to become less accurate from approximatively $50 \%$ on of the total load.

\footnotetext{
${ }^{1}$ Results from $[50,49,53]$ have been digitalized from the papers by the author; therefore they might be affected by some approximations.
} 


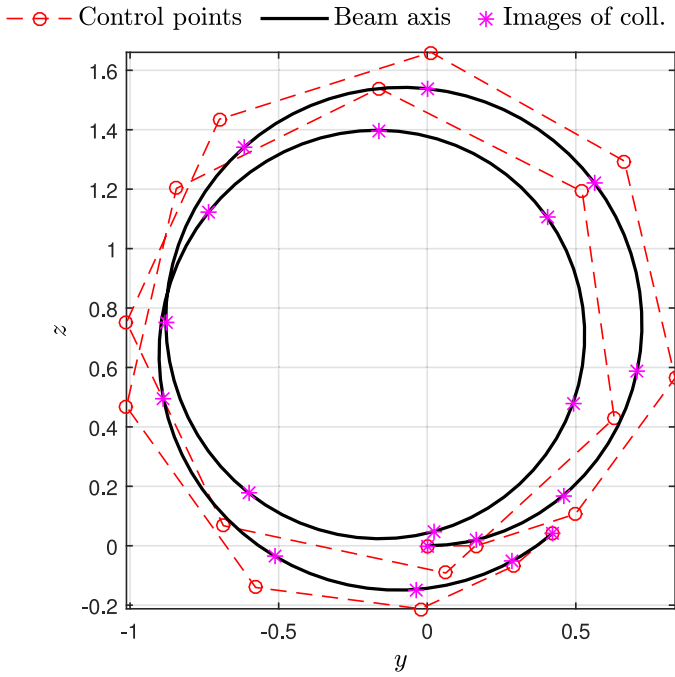

(a) $p=4, n=18$.

$-\ominus-$ Control points - Beam axis * Images of coll. points

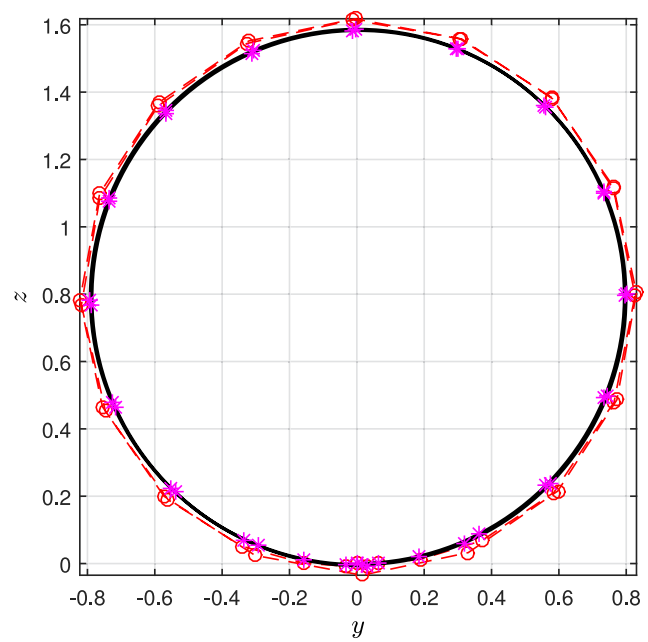

(c) $p=5, n=36$.

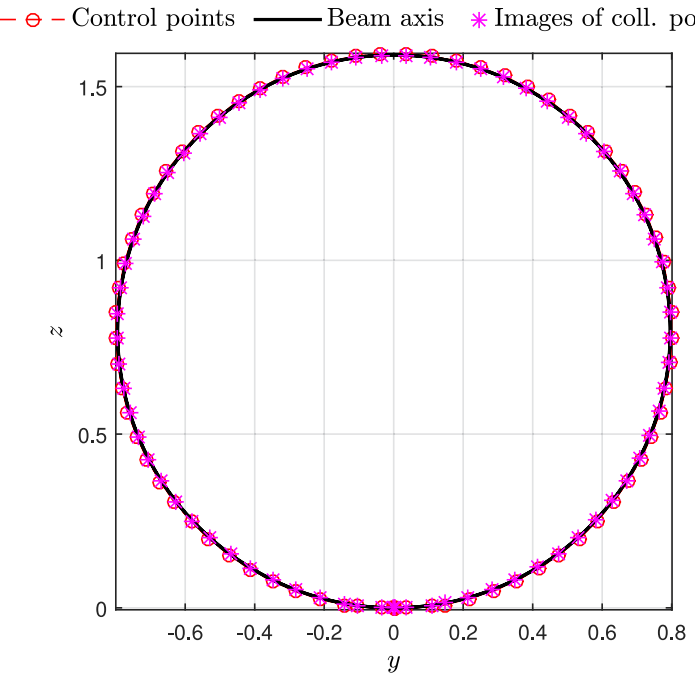

(b) $p=4, n=72$.

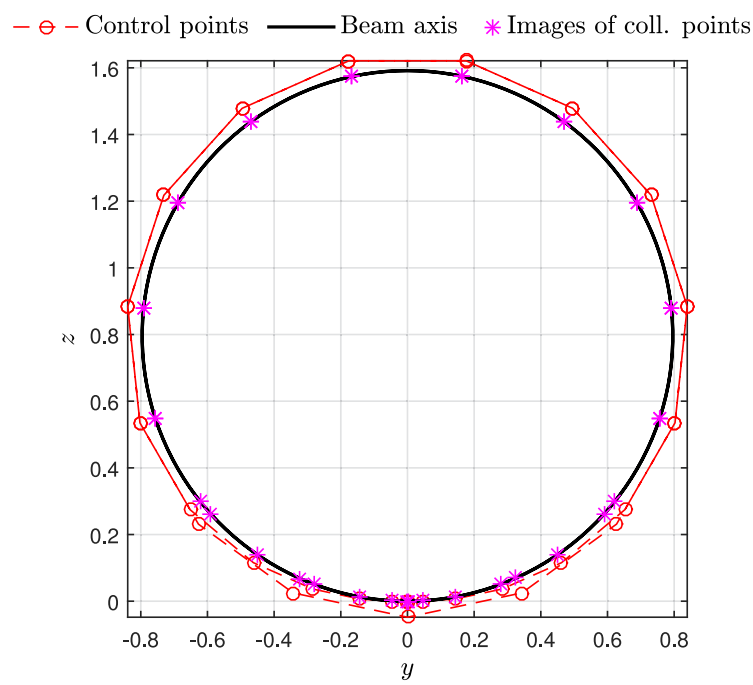

(d) $p=7, n=36$.

Fig. 5. Double-circle test: an example of $n$ refinement (a) and (b) and $p$ refinement (c) and (d). Frontal view of the deformed beam at final configuration.

In addition to $n=80$ (case shown in Fig. 9), we have repeated the same test with $n=90$ and $n=100$. No significant difference in the plot shown in Fig. 9 appeared-all the three curves were almost coincident.

\subsection{Locking study}

With this numerical test we intend to provide a preliminary assessment of the sensitivity of the proposed numerical scheme to the phenomenon of shear locking and compare it with standard FE formulations for geometrically nonlinear beams.

Within the FE method framework, comparisons of different geometrically nonlinear beam formulations in terms of locking are discussed in [64] and a locking-free hybrid formulation is presented in [65]. Both papers confirm that the standard geometrically exact two-node displacement-based finite element formulation leads to severe locking, unless a one-point Gaussian integration rule is used. This strategy, see e.g. [66], represents the most common shear locking countermeasure used in geometrically exact beam formulations. 

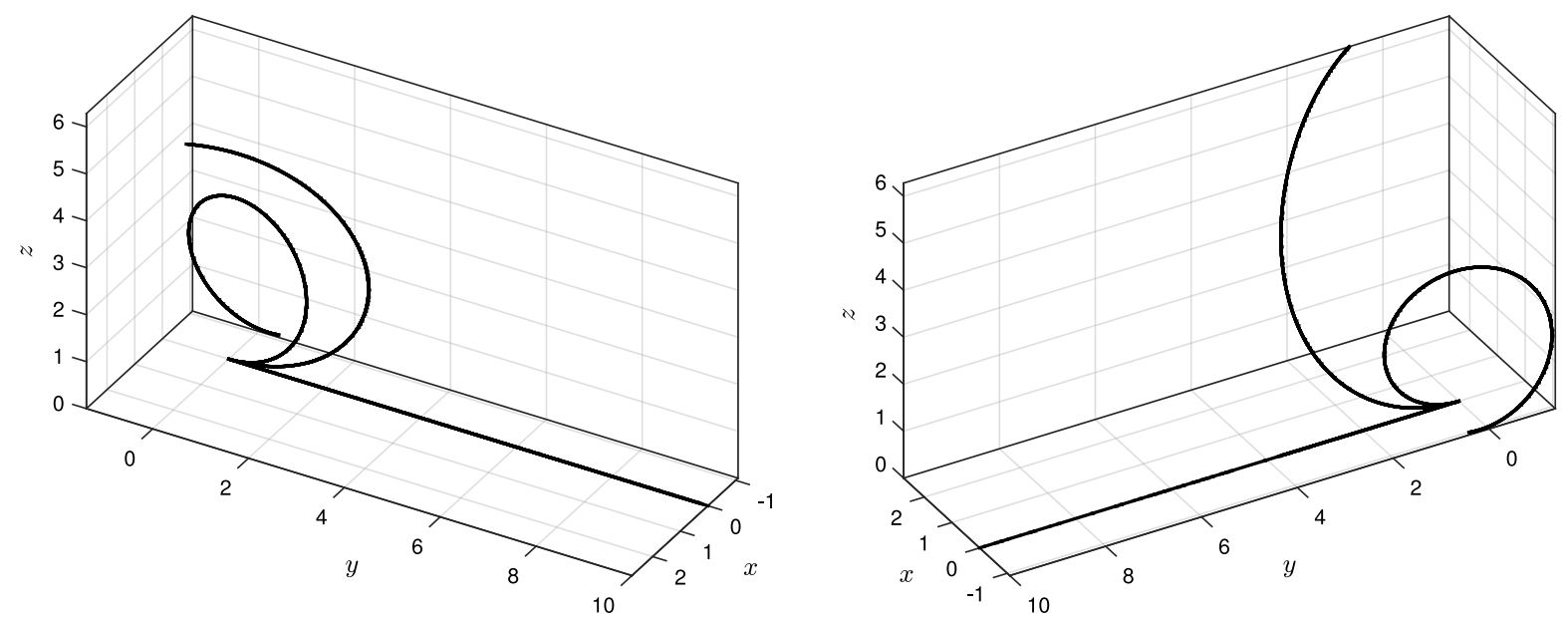

Fig. 6. Helical beam test: two different three-dimensional views of the configurations at $0 \%, 5 \%$ and $10 \%$ of the final loads.

We repeat the same test proposed in [65] that consists of a cantilever beam of length $L=1$ subjected to a lateral concentrated tip load $f=10^{-5}$. The beam cross section width is 0.01 and the slenderness $L / h$, where $h$ denotes the beam thickness, varies from 1.25 up to 1000 . Young's modulus is $E=10^{6}$ and Poisson's ratio is 0.3 .

The dimensionless tip displacements $\delta / \delta_{\text {ref }}$ vs. the beam slenderness is shown in Fig. 10 . $\delta_{\text {ref }}$ indicates the reference solutions, which are obtained using the exact formula in the linear cases and, in the nonlinear case, a geometrically exact FE solver with 40 two-node elements with one-point Gaussian integration scheme to avoid shear locking. The tip displacements $\delta$ for the six beams are computed using the present model with different basis function degrees $(p=3,4,6)$ and two levels of mesh size: $n=10,40$.

We observe that for all approximation degrees and number of collocation points considered, up to the slenderness ratio of 200, shear locking does not appear. For more slender beams, when low approximation degree is used $(p=3)$, by increasing the number of collocation points from 10 to 40, no benefit is seen on the tip displacement. This clearly shows the presence of shear locking. On the contrary, with higher basis function degrees $(p=4,6)$ the reference solution is retrieved when the number of collocation points is increased. For $p=4,6$, up to $L / h=200$, the same results as in Fig. 10 (not shown here) are obtained starting with a minimum value of $n=4$.

We remark that a similar poor behavior of low degree approximation has also been observed in [27,28] for the linear regime. Therefore, in future studies, we might expect that the same remedies against shear locking proposed in $[27,28]$, e.g. mixed formulations, might be effective also in the case of geometrical nonlinearities.

In Fig. 10 we report also the tip deflections digitized from Fig. 4 of [65] (see black solid line marked with triangles) obtained using a standard two-node displacement-based total Lagrangian finite element formulation with five-point Gaussian integration scheme. The deflections tend to zero when the slenderness increases, indicating severe shear locking.

\section{Conclusions}

In this paper we have made a first step towards the extension of isogeometric collocation methods to geometrically exact rods. The definition of beam and the associated kinematics are formulated in such a way that the concept of moving frame is not necessary. The strong form of the displacement-based governing equations is fully linearized consistently with the underlying geometric structure of the configuration manifold. The linearized equations are discretized with NURBS basis functions and collocated at the Greville abscissa. The rotation operator is updated by superimposing the incremental rotation to the current rotation within a consistent incremental-iterative solution scheme. Incremental rotations are parametrized through Eulerian rotation vectors which, together with the beam axis displacement vectors, represent the primary kinematic variables. The update operation is performed through the exponential map.

The proposed combination of IGA-C with the chosen finite rotations parametrization results computationally efficient and has the following main advantages: (i) low computational cost insured by the collocation method and 


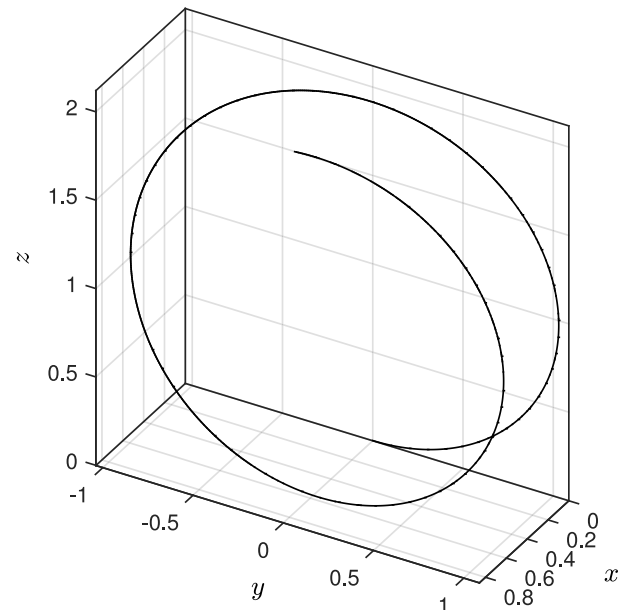

(a) $15 \%$ of final loads.

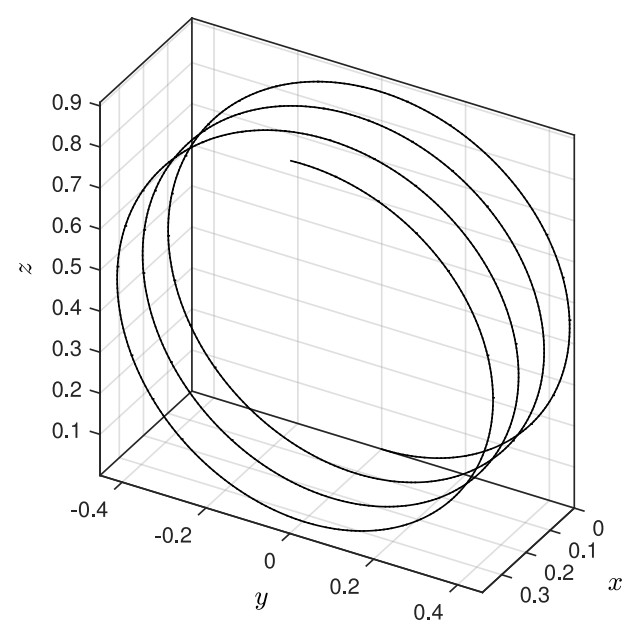

(c) $35 \%$ of final loads.

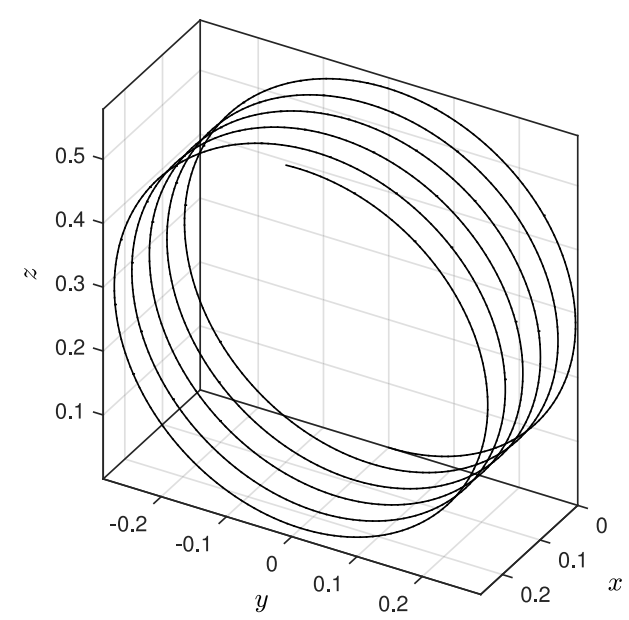

(e) $55 \%$ of final loads.

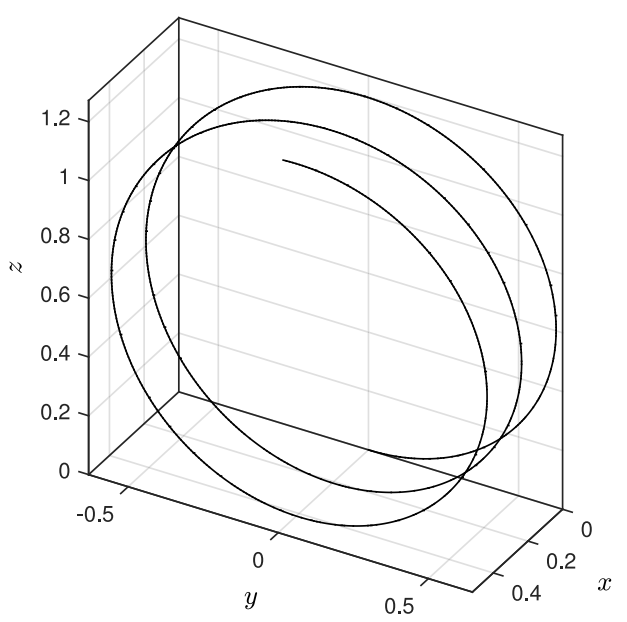

(b) $25 \%$ of final loads.

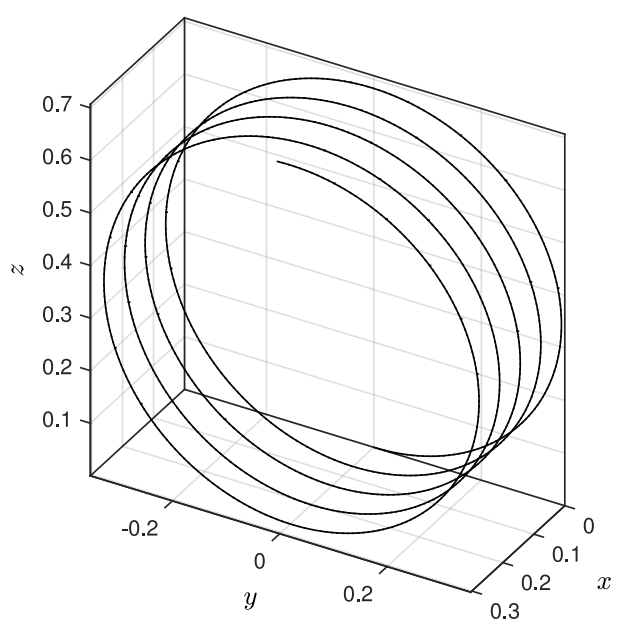

(d) $45 \%$ of final loads.

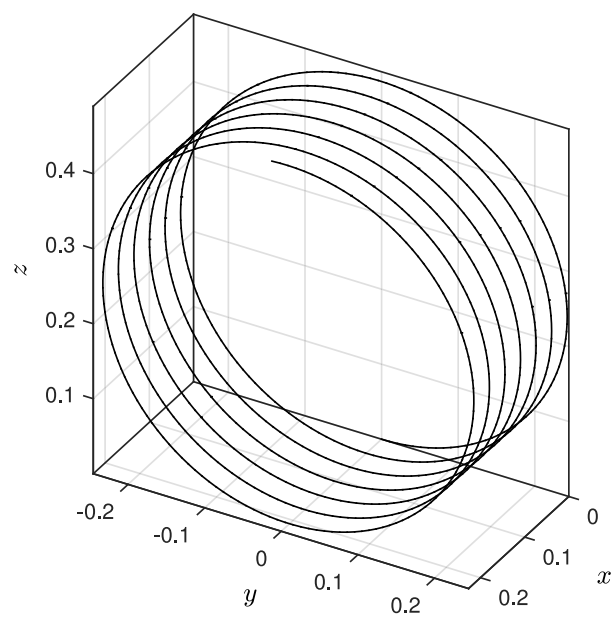

(f) $65 \%$ of final loads.

Fig. 7. Helical beam test: configurations of the deformed beam for load levels from $15 \%$ to $65 \%$ of the final loads. 


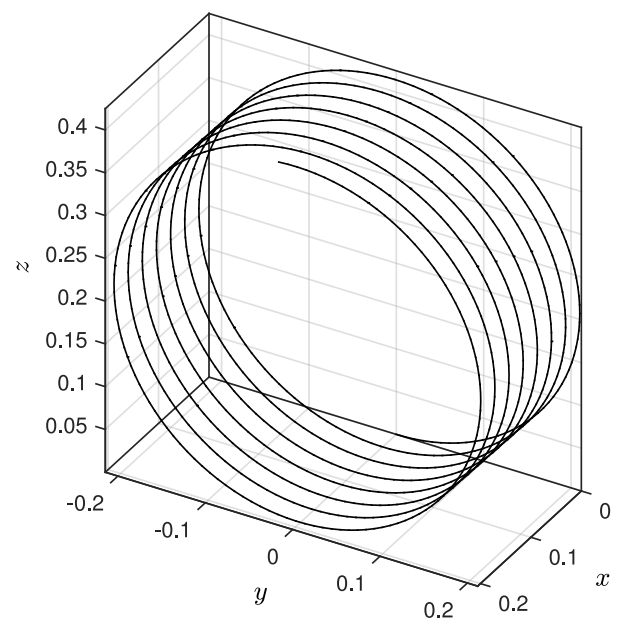

(a) $75 \%$ of final loads.

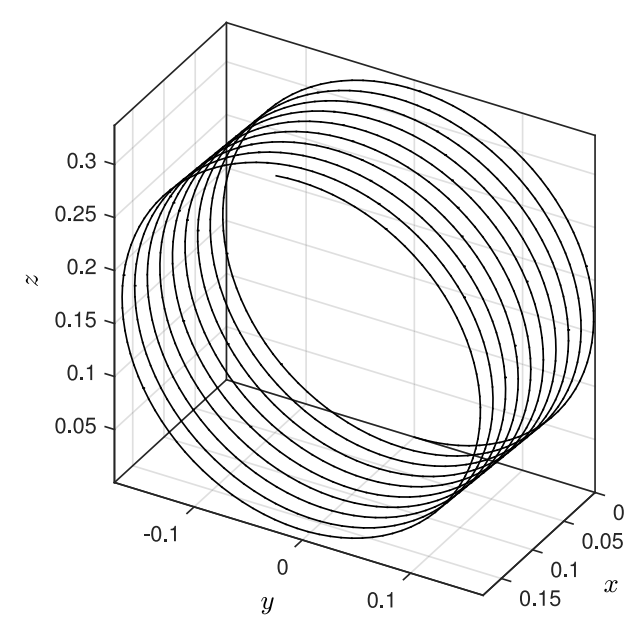

(c) $95 \%$ of final loads.

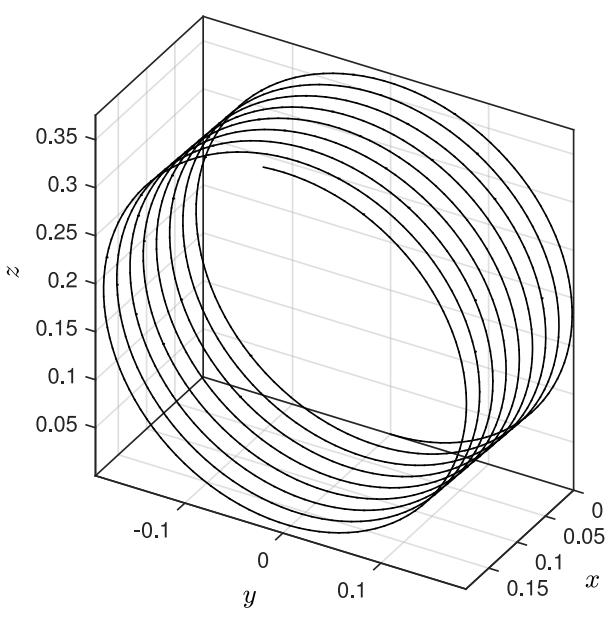

(b) $85 \%$ of final loads.

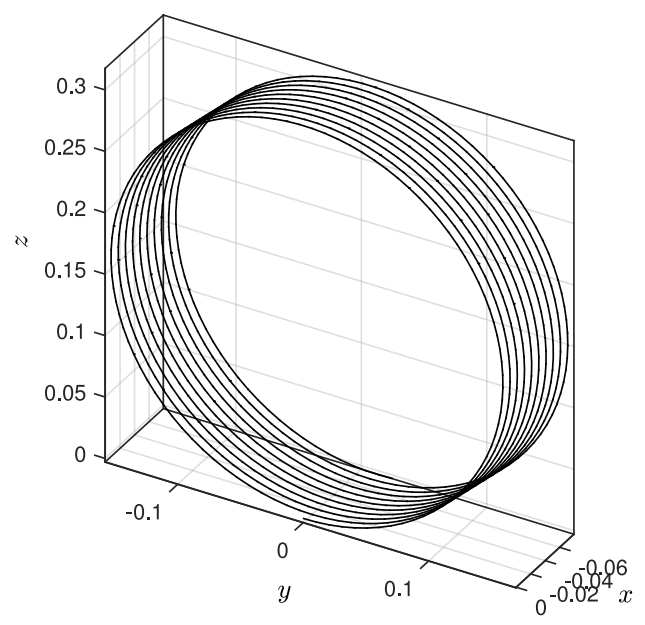

(d) $100 \%$ of final loads.

Fig. 8. Helical beam test: configurations of the deformed beam for load levels from $75 \%$ to $100 \%$ of the final loads.

(ii) capability of handling very large rotations without incurring singularity problems insured by the Eulerian updating scheme. Moreover, the formulation is simple and can be implemented straightforwardly avoiding the linearization of additional geometric operators, such as the tangential transformation which requires cumbersome calculations. The variational formulation is totally avoided implying simplifications in the theoretical formulation.

Numerical tests have shown excellent spatial convergence rates for rotations of any magnitude. Comparisons with FE approaches based on both total Lagrangian formulations (total vector-like parametrization) and incremental vector-like parametrization have proven that the present model can handle complex three-dimensional problems with high accuracy levels. Moreover, a preliminary study has shown that low approximation degree suffers shear locking. However, a comparison with literature results on geometrically exact beams reveals that the present displacementbased IGA-C formulation appears to be naturally much less sensitive than standard FEM to shear locking when reduced order integration schemes are not used.

Isogeometric collocation appears to be a promising approximation technique for geometrical nonlinear beams. A number of important aspects, such as frame invariance, path-dependency, further studies on shear locking, mixed formulations, different choices of collocation points, different parametrizations of finite rotations still need to be investigated for a full extension of IGA-C to geometrically exact beam formulations. 


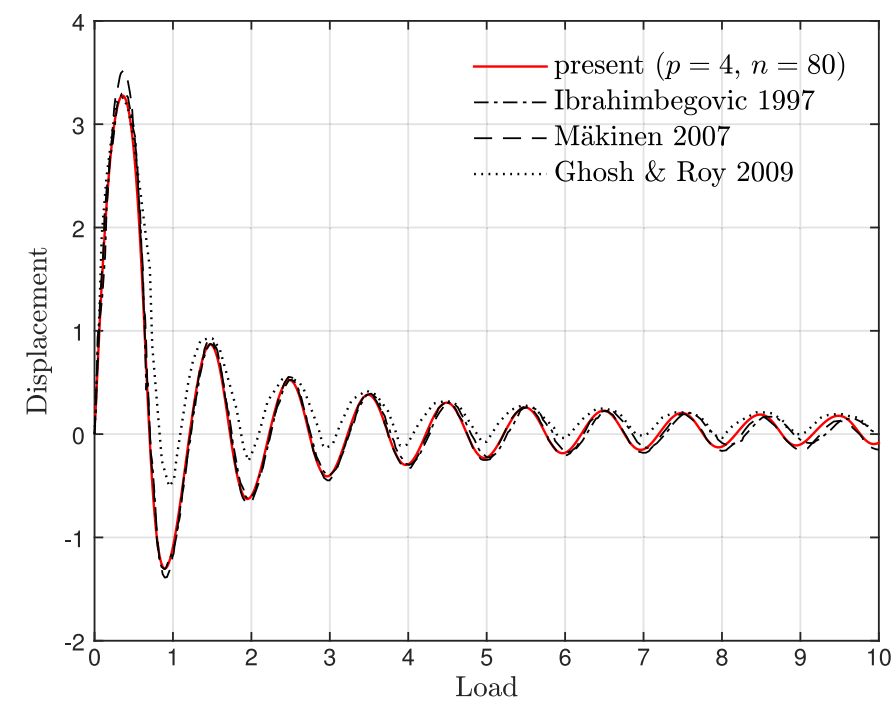

Fig. 9. Helical beam test: comparison of the out-of-plane beam tip displacement between Ibrahimbegovic 1997 [50] (black dashed-dotted line), Mäkinen 2007 [49] (black dashed line), Ghosh and Roy 2009 [53] (dotted line), and present model (red solid line). (For interpretation of the references to colour in this figure legend, the reader is referred to the web version of this article.)

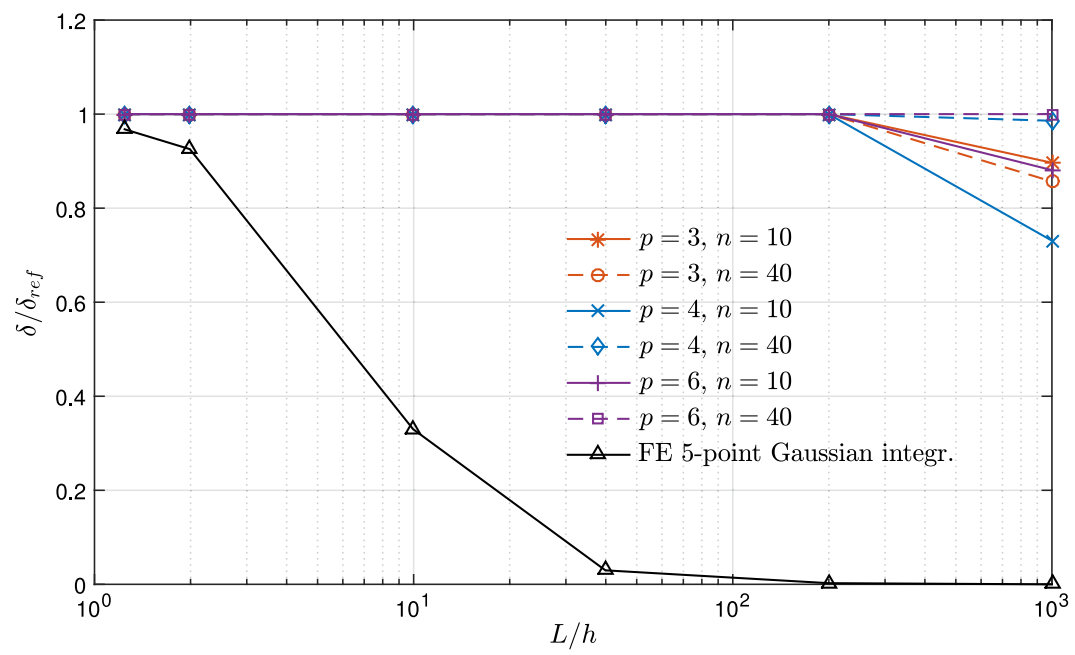

Fig. 10. Effect of slenderness on the tip deflections for different combinations of basis function degrees and number of collocation points. The solid black line marked with triangles is from Fig. 4 of Santos et al. 2011 [65].

\section{Acknowledgments}

I wish to express my sincere gratitude to Prof. Claudio Lugni, Prof. Marco Modugno, Prof. Laura De Lorenzis, and Prof. Josef Kiendl for the helpful discussions. I am also thankful to Lapo Gori for his contribution in computing reference data used in Fig. 10.

\section{Appendix A}

In this Appendix we calculate all the directional derivatives needed for the linearization of the displacement-based governing equations given in Section 5.

Directional derivative of $c,{ }_{s}$.

$$
\left.\frac{d}{d \varepsilon} c_{\varepsilon, s}\right|_{\varepsilon=0}=\frac{d}{d \varepsilon}\left(c_{\varepsilon, s}\right)_{\varepsilon=0}=\boldsymbol{\eta}, s .
$$


Directional derivative of $c,{ }_{s s}$.

$$
\frac{d}{d \varepsilon}\left(c_{\varepsilon, s s}\right)_{\varepsilon=0}=\eta, s s .
$$

Directional derivative of $\mathbf{R}$.

$$
\frac{d}{d \varepsilon}\left(\mathbf{R}_{\varepsilon}\right)_{\varepsilon=0}=\tilde{\vartheta} \mathbf{R}
$$

Directional derivative of $\mathbf{R}^{\top}$. First, by recalling that

$$
\exp (\varepsilon \tilde{\boldsymbol{\vartheta}}(t, q))^{\top}=\exp (-\varepsilon \tilde{\boldsymbol{\vartheta}}(t, q)),
$$

we observe that $\mathbf{R}_{\varepsilon}^{\top}(t, q)=\mathbf{R}^{\top}(t, q) \exp (-\varepsilon \widetilde{\vartheta}(t, q))$. From which we obtain

$$
\frac{d}{d \varepsilon}\left(\mathbf{R}_{\varepsilon}^{\top}\right)_{\varepsilon=0}=-\mathbf{R}^{\top} \tilde{\vartheta}
$$

Directional derivative of $\mathbf{R},{ }_{s}$. First we observe that

$$
\mathbf{R}_{\varepsilon, s}=\exp (\varepsilon \widetilde{\boldsymbol{\vartheta}}),{ }_{s} \mathbf{R}+\exp (\varepsilon \widetilde{\boldsymbol{\vartheta}}) \mathbf{R} \widetilde{\boldsymbol{K}}
$$

where we have used $\mathbf{R}{ }_{s}=\mathbf{R} \widetilde{\boldsymbol{K}}$. Then we obtain the directional derivative as follows

$$
\frac{d}{d \varepsilon}\left(\mathbf{R}_{\varepsilon, s}\right)_{\varepsilon=0}=\tilde{\vartheta},{ }_{s} \mathbf{R}+\tilde{\vartheta} \mathbf{R} \tilde{\boldsymbol{K}}
$$

Directional derivative of $\mathbf{R}^{\top},{ }_{s}$. First we observe that

$$
\mathbf{R}_{\varepsilon, s}^{\top}=\mathbf{R}^{\top}{ }_{s} \exp (-\varepsilon \widetilde{\vartheta})+\mathbf{R}^{\top} \exp (-\varepsilon \widetilde{\vartheta}){ }_{s}
$$

from which we obtain the directional derivative as follows

$$
\frac{d}{d \varepsilon}\left(\mathbf{R}_{\varepsilon, s}^{\top}\right)_{\varepsilon=0}=\widetilde{\boldsymbol{K}} \mathbf{R}^{\top} \tilde{\vartheta}-\mathbf{R}^{\top} \tilde{\vartheta}_{s},
$$

where we have used $\mathbf{R}^{\top}{ }_{s}=-\widetilde{\boldsymbol{K}} \mathbf{R}^{\top}$.

Directional derivative of $\widetilde{\boldsymbol{K}}$. Making use of the above results (A.4) and (A.6), it follows that

$$
\frac{d}{d \varepsilon}\left(\widetilde{\boldsymbol{K}}_{\varepsilon}\right)_{\varepsilon=0}=\frac{d}{d \varepsilon}\left(\mathbf{R}_{\varepsilon}^{\top} \mathbf{R}_{\varepsilon, s}\right)_{\varepsilon=0}=\mathbf{R}^{\top} \widetilde{\vartheta},_{s} \mathbf{R} .
$$

The corresponding axial vector is found considering that

$$
\left(\mathbf{R}^{\top} \widetilde{\vartheta}_{s} \mathbf{R}\right) \boldsymbol{h}=\frac{d}{d \varepsilon}\left(\boldsymbol{K}_{\varepsilon}\right)_{\varepsilon=0} \times \boldsymbol{h}
$$

from which, multiplying both sides by $\mathbf{R}$, we obtain

$$
\boldsymbol{\vartheta},{ }_{s} \times \mathbf{R} \boldsymbol{h}=\mathbf{R} \frac{d}{d \varepsilon}\left(\boldsymbol{K}_{\varepsilon}\right)_{\varepsilon=0} \times \mathbf{R} \boldsymbol{h},
$$

which leads to the following directional derivative of the curvature vector

$$
\frac{d}{d \varepsilon}\left(\boldsymbol{K}_{\varepsilon}\right)_{\varepsilon=0}=\mathbf{R}^{\top} \boldsymbol{\vartheta},_{s} .
$$

Directional derivative of $\boldsymbol{\Gamma}$. Making use of the above results (A.1) and (A.4), it follows that

$$
\frac{d}{d \varepsilon}\left(\boldsymbol{\Gamma}_{\varepsilon}\right)_{\varepsilon=0}=\frac{d}{d \varepsilon}\left(\mathbf{R}_{\varepsilon}^{\top} c_{\varepsilon, s}-\mathbf{n}\right)_{\varepsilon=0}=\mathbf{R}^{\top}\left(\boldsymbol{\eta},,_{s}-\vartheta \times c,_{s}\right) .
$$


Directional derivative of $\boldsymbol{\Gamma},{ }_{s}$. First we observe that

$$
\boldsymbol{\Gamma}_{\varepsilon, s}=\mathbf{R}_{\varepsilon, s}^{\top} c_{\varepsilon, s}+\mathbf{R}_{\varepsilon}^{\top} c_{\varepsilon, s s},
$$

from which, by recalling Eqs. (A.8), (A.1), (A.4), (A.2), we obtain the directional derivative as follows

$$
\frac{d}{d \varepsilon}\left(\boldsymbol{\Gamma}_{\varepsilon, s}\right)_{\varepsilon=0}=\widetilde{\boldsymbol{K}} \mathbf{R}^{\top} \widetilde{\boldsymbol{\vartheta}} c,{ }_{s}-\mathbf{R}^{\top} \widetilde{\boldsymbol{\vartheta}}{ }_{s} c,,_{s}-\widetilde{\boldsymbol{K}} \mathbf{R}^{\top} \boldsymbol{\eta},{ }_{s}-\mathbf{R}^{\top} \widetilde{\boldsymbol{\vartheta}} c,_{s s}+\mathbf{R}^{\top} \boldsymbol{\eta},{ }_{s s}
$$

Directional derivative of $\mathbf{R},{ }_{s s}$. First we observe that

$$
\mathbf{R}_{\varepsilon, s s}=\exp (\varepsilon \widetilde{\vartheta}){ }_{s s} \mathbf{R}+2 \exp (\varepsilon \widetilde{\boldsymbol{\vartheta}}),{ }_{s} \mathbf{R} \widetilde{\boldsymbol{K}}+\exp (\varepsilon \widetilde{\boldsymbol{\vartheta}}) \mathbf{R} \widetilde{\boldsymbol{K}}^{2}+\exp (\varepsilon \widetilde{\boldsymbol{\vartheta}}) \mathbf{R} \widetilde{\boldsymbol{K}},_{s},
$$

where we have used $\mathbf{R},{ }_{s}=\mathbf{R} \widetilde{\boldsymbol{K}}$ and $\mathbf{R},{ }_{s s}=(\mathbf{R} \widetilde{\boldsymbol{K}}){ }_{s}=\mathbf{R}\left(\widetilde{\boldsymbol{K}}^{2}+\widetilde{\boldsymbol{K}},_{s}\right)$. Then we obtain the directional derivative as follows

$$
\frac{d}{d \varepsilon}\left(\mathbf{R}_{\varepsilon, s s}\right)_{\varepsilon=0}=\widetilde{\boldsymbol{\vartheta}},_{s s} \mathbf{R}+2 \widetilde{\boldsymbol{\vartheta}},_{s} \mathbf{R} \widetilde{\boldsymbol{K}}+\widetilde{\vartheta} \mathbf{R}\left(\widetilde{\boldsymbol{K}}^{2}+\widetilde{\boldsymbol{K}},_{s}\right)
$$

Directional derivative of $\boldsymbol{K},{ }_{s}$. We start with the derivative of $\widetilde{\boldsymbol{K}},_{s}$ and recall that

$$
\widetilde{\boldsymbol{K}}_{\varepsilon, s}=\left(\mathbf{R}_{\varepsilon}^{\top} \mathbf{R}_{\varepsilon, s}\right),_{s}=\mathbf{R}_{\varepsilon, s}^{\top} \mathbf{R}_{\varepsilon, s}+\mathbf{R}_{\varepsilon}^{\top} \mathbf{R}_{\varepsilon, s s}
$$

from which, by making use of Eqs. (A.8), (A.6), (A.4), and (A.17), and after some manipulations, we obtain

$$
\frac{d}{d \varepsilon}\left(\widetilde{\boldsymbol{K}}_{\varepsilon, s}\right)_{\varepsilon=0}=\mathbf{R}^{\top} \widetilde{\vartheta},_{s} \mathbf{R} \widetilde{\boldsymbol{K}}-\widetilde{\boldsymbol{K}} \mathbf{R}^{\top} \widetilde{\boldsymbol{\vartheta}},_{s} \mathbf{R}+\mathbf{R}^{\top} \widetilde{\boldsymbol{\vartheta}},_{s s} \mathbf{R}
$$

Inspecting Eq. (A.19), and by recalling the identity $(\widetilde{\boldsymbol{a}} \widetilde{\boldsymbol{b}}-\widetilde{\boldsymbol{b}} \widetilde{\boldsymbol{a}}) \boldsymbol{h}=(\boldsymbol{a} \times \boldsymbol{b}) \times \boldsymbol{h}$, where $\boldsymbol{a}$ and $\boldsymbol{b}$ are the axial vectors of the associated skew-symmetric tensors $\widetilde{\boldsymbol{a}}, \widetilde{\boldsymbol{b}} \in \operatorname{so}(3)$ for any $\boldsymbol{h} \in \mathbb{R}^{3}$, we finally recognize that

$$
\frac{d}{d \varepsilon}\left(\boldsymbol{K}_{\varepsilon, s}\right)_{\varepsilon=0}=\mathbf{R}^{\top} \tilde{\vartheta}_{, s} \mathbf{R} \boldsymbol{K}+\mathbf{R}^{\top} \boldsymbol{\vartheta}, s s
$$

where we have also used the fact that $\mathbf{R}^{\top} \vartheta{ }_{s s}$ is the axial vector of $\mathbf{R}^{\top} \widetilde{\vartheta},{ }_{s s} \mathbf{R}$ as observed previously in the derivation of the directional derivative of $\widetilde{\boldsymbol{K}}$.

\section{Appendix B}

\section{Derivative of the exponential map}

As discussed in Section 7, once the primary kinematic unknowns $\left(\breve{\boldsymbol{\vartheta}}_{i}^{(k)}, \check{\boldsymbol{\eta}}_{i}^{(k)}\right)$ with $i=0, \ldots, n$ are computed at the $k$ th iteration, all the kinematic quantities need to be consistently updated in order to form the $(k+1)$ th system matrix. We emphasize that the overall quality of the convergence rate in the Newton algorithm much depends on the accuracy used to update the curvature tensor and its derivative, see Eqs. (63) and (64) respectively. These updating formulas involve the first and second derivative of the exponential map. In this section, we provide a practical approach that can be used to approximate, up to the desired order, these derivatives.

Consider a curve in so(3), $s \mapsto \widetilde{\vartheta}(s) \in \operatorname{so}(3), s \in \mathcal{I}_{s} \subset \mathbb{R}$. As usual, we denote by $\widetilde{\vartheta}$ the skew-symmetric tensor associated with the vector $\vartheta$. By the chain rule we have

$$
\frac{d}{d s} \exp (\widetilde{\vartheta}(s))=\exp (\widetilde{\vartheta})(d \exp \tilde{\vartheta}) \widetilde{\vartheta}_{s}
$$

where the derivative of the exponential map with respect to $\widetilde{\vartheta}$, see for example [67, p. 15], is given by

$$
d \exp \widetilde{\vartheta}=\frac{1-\exp (-\operatorname{ad} \widetilde{\vartheta})}{\operatorname{ad} \widetilde{\vartheta}}=\sum_{i=0}^{\infty} \frac{(-1)^{i}}{(1+i) !}(\operatorname{ad} \widetilde{\vartheta})^{i}
$$


In Eq. (B.2), the operator ad is the adjoint representation of the Lie algebra so(3) on itself and it is defined as

$$
\operatorname{ad}: \operatorname{so}(3) \rightarrow L(\operatorname{so}(3), \operatorname{so}(3)): \widetilde{\vartheta} \mapsto \operatorname{ad} \widetilde{\vartheta}:=[\widetilde{\vartheta}, \cdot],
$$

where $L(\operatorname{so}(3)$, so(3)) is the space of linear maps in the Lie algebra and $[\cdot, \cdot]$ denotes the Lie brackets, see for example [42, p. 167] for additional details. With these tools at hand we are able to express Eq. (B.1) by expanding the series as follows

$$
\begin{aligned}
\frac{d}{d s} \exp (\tilde{\boldsymbol{\vartheta}}(s)) & =\exp (\tilde{\boldsymbol{\vartheta}})\left(\sum_{i=0}^{\infty} \frac{(-1)^{i}}{(1+i) !}(\operatorname{ad} \tilde{\boldsymbol{\vartheta}})^{i}\right) \tilde{\boldsymbol{\vartheta}},,_{s} \\
& =\exp (\tilde{\boldsymbol{\vartheta}})\left(\tilde{\boldsymbol{\vartheta}},_{s}-\frac{1}{2}\left[\tilde{\boldsymbol{\vartheta}}, \tilde{\boldsymbol{\vartheta}},_{s}\right]+\frac{1}{6}\left[\tilde{\boldsymbol{\vartheta}},\left[\tilde{\boldsymbol{\vartheta}}, \tilde{\boldsymbol{\vartheta}},{ }_{s}\right]\right]-\frac{1}{24}\left[\tilde{\boldsymbol{\vartheta}},\left[\tilde{\boldsymbol{\vartheta}},\left[\tilde{\boldsymbol{\vartheta}}, \tilde{\boldsymbol{\vartheta}},,_{s}\right]\right]+\cdots\right)\right.
\end{aligned}
$$

We observe that keeping only the zero-order term of the series would mean assuming that $\left[\widetilde{\boldsymbol{\vartheta}}, \widetilde{\vartheta},{ }_{s}\right]=0$. This amounts to consider the vector $\vartheta$ and $\vartheta, s$ having the same direction. This is not generally true, excepting for the special case of two-dimensional motions, where rotations occur always around a fixed axis. Such a circumstance represents the special case for which rotations are commutative.

We also point out that the above formula is computationally efficient due to the possibility to easily compute nested Lie brackets up to the required order.

Proof of the updating formulas (Eqs. (63) and (64))

We start with the proof of Eq. (63). By definition of curvature tensor, we have

$$
\begin{aligned}
& \widetilde{\boldsymbol{K}}^{(k+1)}=\mathbf{R}^{\boldsymbol{\top}^{(k+1)}} \mathbf{R},{ }_{s}^{(k+1)}=\left(\exp \left(\widetilde{\vartheta}^{(k)}\right) \mathbf{R}^{(k)}\right)^{\top}\left(\exp \left(\widetilde{\vartheta}^{(k)}\right) \mathbf{R}^{(k)}\right), s \\
& =\left(\mathbf{R}^{\mathbf{T}^{(k)}} \exp \left(-\tilde{\vartheta}^{(k)}\right)\right)\left(\exp \left(\widetilde{\vartheta}^{(k)}\right){ }_{s} \mathbf{R}^{(k)}+\exp \left(\widetilde{\vartheta}^{(k)}\right) \mathbf{R},{ }_{s}^{(k)}\right) \\
& =\mathbf{R}^{\boldsymbol{T}^{(k)}} \exp \left(-\widetilde{\boldsymbol{\vartheta}}^{(k)}\right) \exp \left(\widetilde{\boldsymbol{\vartheta}}^{(k)}\right){ }_{s} \mathbf{R}^{(k)}+\widetilde{\boldsymbol{K}}^{(k)} .
\end{aligned}
$$

By substituting (B.4) into the above derivation we get the sought updating formula. We note that instead of the series (B.4), an exact formula for updating the curvature tensor can alternatively be used [40,68].

Following the same procedure (omitted here), and making use of (B.2), Eq. (64) is obtained. The second derivative of the exponential map ( $\left.d \exp _{\widetilde{\vartheta}} \widetilde{\vartheta}{ }_{,}^{(k)}\right)$, ${ }_{s}$ appearing in Eq. (64) is computed by direct differentiation of the above series. The axial vector associated with the curvature tensor derivative is obtained from Eq. (64) by exploiting again the identity $(\widetilde{\boldsymbol{a}} \widetilde{\boldsymbol{b}}-\widetilde{\boldsymbol{b}} \widetilde{\boldsymbol{a}}) \boldsymbol{h}=(\boldsymbol{a} \times \boldsymbol{b}) \times \boldsymbol{h}$, with $\widetilde{\boldsymbol{a}}, \widetilde{\boldsymbol{b}} \in \operatorname{so}(3)$ and $\boldsymbol{h} \in \mathbb{R}^{3}$.

\section{References}

[1] T. Hughes, J. Cottrell, Y. Bazilevs, Isogeometric analysis: CAD, finite elements, NURBS, exact geometry and mesh refinement, Comput. Methods Appl. Mech. Engrg. 194 (39-41) (2005) 4135-4195.

[2] J.A. Cottrell, T.J.R. Hughes, Y. Bazilevs, Isogeometric Analysis: Toward Integration of CAD and FEA, JohnWiley \& Sons, Ltd Registered, 2009.

[3] J. Cottrell, A. Reali, Y. Bazilevs, T. Hughes, Isogeometric analysis of structural vibrations, Comput. Methods Appl. Mech. Engrg. 195 (41-43) (2006) 5257-5296.

[4] A. Reali, An isogeometric analysis approach for the study of structural vibrations, J. Earthq. Eng. 10 (2006) 1.

[5] J. Kiendl, K.-U. Bletzinger, J. Linhard, R. Wüchner, Isogeometric shell analysis with Kirchhoff-Love elements, Comput. Methods Appl. Mech. Engrg. 198 (49-52) (2009) 3902-3914.

[6] D. Benson, Y. Bazilevs, M.-C. Hsu, T. Hughes, A large deformation, rotation-free, isogeometric shell, Comput. Methods Appl. Mech. Engrg. 200 (13-16) (2011) 1367-1378.

[7] R. Bouclier, T. Elguedj, A. Combescure, Locking free isogeometric formulations of curved thick beams, Comput. Methods Appl. Mech. Engrg. 245-246 (2012) 144-162.

[8] L. Greco, M. Cuomo, B-Spline interpolation of Kirchhoff-Love space rods, Comput. Methods Appl. Mech. Engrg. 256 (2013) $251-269$.

[9] T. Elguedj, T. Hughes, Isogeometric analysis of nearly incompressible large strain plasticity, Comput. Methods Appl. Mech. Engrg. 268 (2014) 388-416.

[10] R. Dimitri, L. De Lorenzis, M. Scott, P. Wriggers, R. Taylor, G. Zavarise, Isogeometric large deformation frictionless contact using T-splines, Comput. Methods Appl. Mech. Engrg. 269 (2014) 394-414. 
[11] L. De Lorenzis, P. Wriggers, T.J.R. Hughes, Isogeometric contact: a review, GAMM-Mitt. 37 (1) (2014) 85-123.

[12] N. Nguyen-Thanh, N. Valizadeh, M. Nguyen, H. Nguyen-Xuan, X. Zhuang, P. Areias, G. Zi, Y. Bazilevs, L. De Lorenzis, T. Rabczuk, An extended isogeometric thin shell analysis based on Kirchhoff-Love theory, Comput. Methods Appl. Mech. Engrg. 284 (2015) $265-291$.

[13] L. Greco, M. Cuomo, An isogeometric implicit G1 mixed finite element for Kirchhoff space rods, Comput. Methods Appl. Mech. Engrg. 298 (2016) 325-349.

[14] A. Bauer, M. Breitenberger, B. Philipp, R. Wüchner, K.-U. Bletzinger, Nonlinear isogeometric spatial Bernoulli beam, Comput. Methods Appl. Mech. Engrg. 303 (2016) 101-127.

[15] I. Akkerman, Y. Bazilevs, V.M. Calo, T.J.R. Hughes, S. Hulshoff, The role of continuity in residual-based variational multiscale modeling of turbulence, Comput. Mech. 41 (3) (2008) 371-378.

[16] I. Akkerman, Y. Bazilevs, C.E. Kees, M.W. Farthing, Isogeometric analysis of free-surface flow, J. Comput. Phys. 230 (11) (2011) $4137-4152$.

[17] Y. Bazilevs, V.M. Calo, T.J.R. Hughes, Y. Zhang, Isogeometric fluid-structure interaction: Theory, algorithms, and computations, Comput. Mech. 43 (1) (2008) 3-37.

[18] Y. Bazilevs, M. Hsu, I. Akkerman, S. Wright, T. E. T.K. Takizawa, B. Henicke, T. Spielman, 3D simulation of wind turbine rotors at full scale. Part I: Geometry modeling and aerodynamics, Int. J. Numer. Methods Fluids (65) (2011) 207-235.

[19] Y. Bazilevs, M.C. Hsu, M. a. Scott, Isogeometric fluid-structure interaction analysis with emphasis on non-matching discretizations, and with application to wind turbines, Comput. Methods Appl. Mech. Engrg. 249-252 (2012) 28-41.

[20] C. Heinrich, B. Simeon, S. Boschert, A finite volume method on NURBS geometries and its application in isogeometric fluid-structure interaction, Math. Comput. Simulation 82 (9) (2012) 1645-1666.

[21] T. Hughes, A. Reali, G. Sangalli, Efficient quadrature for NURBS-based isogeometric analysis, Comput. Methods Appl. Mech. Engrg. 199 (5-8) (2010) 301-313.

[22] F. Auricchio, F. Calabrò, T. Hughes, A. Reali, G. Sangalli, A simple algorithm for obtaining nearly optimal quadrature rules for NURBS-based isogeometric analysis, Comput. Methods Appl. Mech. Engrg. 249-252 (2012) 15-27.

[23] F. Auricchio, L.B. Da Veiga, T.J.R. Hughes, A. Reali, G. Sangalli, Isogeometric collocation methods, Math. Model. Methods Appl. Sci. 20 (11) (2010) 2075-2107.

[24] A. Reali, T.J.R. Hughes, An introduction to isogeometric collocation methods, in: G. Beer, S. Bordas (Eds.), Isogeometric Methods for Numerical Simulation, Springer Vienna, Vienna, 2015, pp. 173-204. http://dx.doi.org/10.1007/978-3-7091-1843-6_4.

[25] D. Schillinger, J. a. Evans, A. Reali, M. a. Scott, T.J.R. Hughes, Isogeometric collocation: Cost comparison with Galerkin methods and extension to adaptive hierarchical NURBS discretizations, Comput. Methods Appl. Mech. Engrg. 267 (2013) 170-232.

[26] F. Auricchio, L. Beirão da Veiga, T.J.R. Hughes, A. Reali, G. Sangalli, Isogeometric collocation for elastostatics and explicit dynamics, Comput. Methods Appl. Mech. Engrg. 249-252 (2012) 2-14.

[27] L. Beirão da Veiga, C. Lovadina, A. Reali, Avoiding shear locking for the Timoshenko beam problem via isogeometric collocation methods, Comput. Methods Appl. Mech. Engrg. 241-244 (2012) 38-51.

[28] F. Auricchio, L. Beirão da Veiga, J. Kiendl, C. Lovadina, A. Reali, Locking-free isogeometric collocation methods for spatial Timoshenko rods, Comput. Methods Appl. Mech. Engrg. 263 (2013) 113-126.

[29] A. Reali, H. Gomez, An isogeometric collocation approach for Bernoulli-Euler beams and Kirchhoff plates, Comput. Methods Appl. Mech. Engrg. 284 (2015) 623-636.

[30] J. Kiendl, F. Auricchio, L. Beirão da Veiga, C. Lovadina, A. Reali, Isogeometric collocation methods for the Reissner-Mindlin plate problem, Comput. Methods Appl. Mech. Engrg. 284 (2015) 489-507.

[31] J. Kiendl, F. Auricchio, T. Hughes, A. Reali, Single-variable formulations and isogeometric discretizations for shear deformable beams, Comput. Methods Appl. Mech. Engrg. 284 (2015) 988-1004.

[32] L. De Lorenzis, J. Evans, T. Hughes, A. Reali, Isogeometric collocation: Neumann boundary conditions and contact, Comput. Methods Appl. Mech. Engrg. 284 (2015) 21-54.

[33] R. Kruse, N. Nguyen-Thanh, L. De Lorenzis, T. Hughes, Isogeometric collocation for large deformation elasticity and frictional contact problems, Comput. Methods Appl. Mech. Engrg. 296 (2015) 73-112.

[34] J.C. Simo, A finite strain beam formulation. The three-dimensional dynamic problem. Part I, Comput. Methods Appl. Mech. Engrg. 49 (1) (1985) 55-70.

[35] E. Reissner, On one-dimensional finite-strain beam theory: The plane problem, Z. Angew. Math. Mech. Angew. Math. Phys. ZAMP 23 (1972) 795-804.

[36] S. Antman, Kirchhoff's problem for nonlinearly elastic rods, Q. Appl. Math. XXXII (3) (1974) 221-240.

[37] P.M. Naghdi, Finite deformation of elastic rods and shells, in: Finite Deform. Elastic Rods Shells, Proceedings of the IUTAM Symposium on Finite Elasticity, 1981, pp. 47-103.

[38] J. Argyris, An excursion into large rotations, Comput. Methods Appl. Mech. Engrg. 32 (13) (1982) 85-155.

[39] S. Eugster, Geometric Continuum Mechanics and Induced Beam Theories, Vol. 75, Springer, 2015.

[40] L. Simo, J.C. Vu-Quoc, A three-dimensional finite-strain rod model. Part II: Computational aspects, Comput. Methods Appl. Mech. Engrg. 58 (1) (1986) 79-116.

[41] F. Auricchio, P. Carotenuto, A. Reali, On the geometrically exact beam model: A consistent, effective and simple derivation from threedimensional finite-elasticity, Int. J. Solids Struct. 45 (17) (2008) 4766-4781.

[42] Y. Choquet-Bruhat, C. Dewitt-Morette, Analysis, Manifolds and Physics Part I: Basics, Elsevier B.V., 1996.

[43] J.E. Marsden, T.S. Ratiu, Introduction to Mechanics and Symmetry, second ed., in: Texts in Applied Mathematics, Springer, New York, NY, 1999.

[44] J. Mäkinen, Critical study of newmark-scheme on manifold of finite rotations, Comput. Methods Appl. Mech. Engrg. 191 (2001) 817-828.

[45] J. Mäkinen, Rotation manifold SO(3) and its tangential vectors, Comput. Mech. 42 (6) (2008) 907-919.

[46] A. Cardona, M. Geradin, A beam finite element non-linear theory with finite rotations, Internat. J. Numer. Methods Engrg. 26 (1988) 2403-2438. September 1987. 
[47] A. Ibrahimbegović, F. Frey, I. Kožar, Computational aspects of vector-like parametrization of three-dimensional finite rotations, Internat. J. Numer. Methods Engrg. 38 (21) (1995) 3653-3673.

[48] M. Ritto-Corrêa, D. Camotim, On the differentiation of the Rodrigues formula and its significance for the vector-like parameterization of Reissner-Simo beam theory, Internat. J. Numer. Methods Engrg. 55 (9) (2002) 1005-1032.

[49] J. Mäkinen, Total Lagrangian Reissner's geometrically exact beam element without singularities, Internat. J. Numer. Methods Engrg. 70 (2007) 1009-1048. October 2006.

[50] A. Ibrahimbegovic, On the choice of finite rotation parameters, Comput. methods Appl. Mech. Eng. 149 (3) (1997) 49-71.

[51] H. Cheng, K.C. Gupta, An historical note on finite rotations, J. Appl. Mech. 56 (1) (1989) 139.

[52] A. Ibrahimbegovic, On finite element implementation of geometrically nonlinear Reissner's beam theory: three-dimensional curved beam elements, Comput. Methods Appl. Mech. Eng. 122 (1995) 11-26.

[53] S. Ghosh, D. Roy, A frame-invariant scheme for the geometrically exact beam using rotation vector parametrization, Comput. Mech. 44 (1) (2009) 103-118.

[54] A. Ibrahimbegović, H. Shakourzadeh, J.-L. Batoz, M. AI Mikdad, Y.-Q. Guo, On the role of geometrically exact and second-order theories in buckling and post-buckling analysis of three-dimensional beam structures, Comput. Struct. 61 (6) (1996) 1101-1114.

[55] A. Ibrahimbegović, M. Al Mikdad, Quadratically convergent direct calculation of critical points for 3d structures undergoing finite rotations, Comput. Methods Appl. Mech. Engrg. 189 (1) (2000) 107-120.

[56] A. Ibrahimbegovic, R.L. Taylor, On the role of frame-invariance in structural mechanics models at finite rotations, Comput. Methods Appl. Mech. Engrg. 191 (45) (2002) 5159-5176.

[57] J.E. Marsden, T.J.R. Hughes, Mathematical Foundations Of Elasticity, Dover Publications, Inc., 1994.

[58] G.A. Holzapfel, Nonlinear Solid Mechanics, John Wiley \& Sons, Ltd, 2000.

[59] T.J. Hughes, K.S. Pister, Consistent linearization in mechanics of solids and structures, Comput. Struct. 8 (34) (1978) $391-397$.

[60] M. Epstein, Differentiable manifolds and the principle of virtual work in continuum mechanics, J. Math. Phys. 21 (5) (1980) 1243.

[61] L. Simo, J.C. Vu-Quoc, On the dynamics in space of rods undergoing large motions — A geometrically exact approach, Comput. Methods Appl. Mech. Engrg. 66 (2) (1988) 125-161.

[62] J. Stuelpnagel, On the parametrization of the three-dimensional rotation group, SIAM Rev. 6 (4) (1964) $422-430$.

[63] L. Piegl, W. Tiller, The NURBS Book, Springer, 1997.

[64] I. Romero, A comparison of finite elements for nonlinear beams: the absolute nodal coordinate and geometrically exact formulations, Multibody Syst. Dyn. 20 (1) (2008) 51-68.

[65] H.A.F.A. Santos, P.M. Pimenta, J.P.M. Almeida, A hybrid-mixed finite element formulation for the geometrically exact analysis of threedimensional framed structures, Comput. Mech. 48 (5) (2011) 591-613.

[66] T.J.R. Hughes, The Finite Element Method: Linear Static and Dynamic Finite Element Analysis, Dover Publications, 2000.

[67] W. Rossmann, Lie groups An Introduction Through Linear Groups, oxford gra ed., Oxford University Press, 2002.

[68] G. Jelenić, M. Saje, A kinematically exact space finite strain beam model — finite element formulation by generalized virtual work principle, Comput. Methods Appl. Mech. Engrg. 120 (1-2) (1995) 131-161. 